ARTICLE

\title{
Fungal kinases and transcription factors regulating brain infection in Cryptococcus neoformans
}

Kyung-Tae Lee (1) 1,6, Joohyeon Hong (10) 1,6, Dong-Gi Lee (10) 1,6, Minjae Lee (1) ${ }^{1}$, Suyeon Cha', Yu-Gyeong Lim (1) ${ }^{1}$,

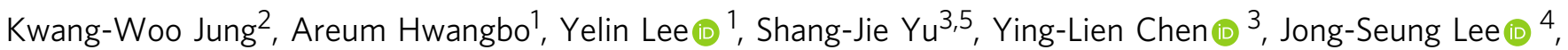
Eunji Cheong (iD) ${ }^{1 \times} \&$ Yong-Sun Bahn (i) ${ }^{1 凶}$

Cryptococcus neoformans causes fatal fungal meningoencephalitis. Here, we study the roles played by fungal kinases and transcription factors (TFs) in blood-brain barrier (BBB) crossing and brain infection in mice. We use a brain infectivity assay to screen signature-tagged mutagenesis (STM)-based libraries of mutants defective in kinases and TFs, generated in the C. neoformans $\mathrm{H} 99$ strain. We also monitor in vivo transcription profiles of kinases and TFs during host infection using NanoString technology. These analyses identify signalling components involved in BBB adhesion and crossing, or survival in the brain parenchyma. The TFs Pdr802, Hob1, and Sre1 are required for infection under all the conditions tested here. Hob1 controls the expression of several factors involved in brain infection, including inositol transporters, a metalloprotease, PDR802, and SRE1. However, Hob1 is dispensable for most cellular functions in Cryptococcus deuterogattii R265, a strain that does not target the brain during infection. Our results indicate that Hob1 is a master regulator of brain infectivity in C. neoformans.

\footnotetext{
${ }^{1}$ Department of Biotechnology, College of Life Science and Biotechnology, Yonsei University, Seoul 03722, Korea. ${ }^{2}$ Research Division for Biotechnology, Korea Atomic Energy Research Institute, Jeongeup 56212, Korea. ${ }^{3}$ Department of Plant Pathology and Microbiology, National Taiwan University, Taipei 10617, Taiwan. ${ }^{4}$ AmtixBio Co., Ltd., Seoul 05836, Korea. ${ }^{5}$ Present address: National Institute of Infectious Diseases and Vaccinology, National Health Research Institute, 35053 Miaoli, Taiwan. ${ }^{6}$ These authors contributed equally: Kyung-Tae Lee, Joohyeon Hong, Dong-Gi Lee. ${ }^{凶}$ email: eunjicheong@yonsei. ac.kr; ysbahn@yonsei.ac.kr
} 
$\mathrm{n}$ the past decades, there has been a growing concern about fungal pathogens that impose a serious threat to both animal and plant ecosystems ${ }^{1}$. A recent epidemiological study has demonstrated that more than 1.5 million people succumb to death due to invasive mycoses ${ }^{2}$. Cryptococcus neoformans is one of the major invasive mycosis-causing opportunistic fungal pathogens, which is responsible for more than 220,000 infections and 180,000 deaths globally every year ${ }^{3}$. Nevertheless, treatment options are highly limited and mortality rate is unacceptably high. Therefore, it is imperative to fully understand the signalling and metabolic networks governing the pathogenicity of $C$. neoformans during host infection for the development of antifungal targets and drugs.

Pathogenic Cryptococcus species complex is widely distributed in a variety of environmental niches ${ }^{4}$. In the natural environment, it generates infectious propagules through heterosexual or unisexual reproduction, which can be inhaled through the respiratory tract of a susceptible mammalian host. At this initial stage of infection, C. neoformans battle against alveolar macrophages residing in the lungs by employing antiphagocytic factors, such as capsule and melanin pigment ${ }^{5-8}$. Even after phagocytosis, the pathogen can survive and proliferate within the phagolysosome and escape from the macrophage, often even without killing the host cell $^{9-11}$. Subsequently, C. neoformans haematogenously disseminates and eventually travels to the central nervous system (CNS), causing meningoencephalitis. Among the infection stages, crossing the blood-brain barrier (BBB) and proliferation in the brain parenchyma are critical factors for $C$. neoformans to impose lethal lesions in mammalian brain tissues. C. neoformans is able to traverse the $\mathrm{BBB}$, which protects the brain from pathogens because of the establishment of tight junctions between brain microvascular endothelial cells (BMECs) and the contribution of brain astrocytes ${ }^{12}$, through transcellular, paracellular, and/or Trojan-horse mechanisms ${ }^{13,14}$. Nevertheless, the complex signalling pathways regulating these known and hitherto uncharacterised factors governing the BBB crossing and brain infection of $C$. neoformans are not completely understood.

To systematically dissect infection-stage dependent signalling networks, here we performed signature-tagged mutagenesis (STM)-based brain infectivity assay with transcription factor (TF) and kinase mutant libraries using intravenous infection route, which can bypass lung infection, and compared these data with that of previously acquired lung-STM data using the same set of mutant libraries but intranasally infected ${ }^{15,16}$. In addition, we performed in vivo transcription profiles of $180 \mathrm{TFs}$ and 183 kinases, along with 58 known virulence-related genes, by using NanoString technology at diverse infected tissues during Cryptococcus infection. For brain-infection-related TFs and kinases, we further analysed their roles in BBB adhesion/crossing and survival within the brain parenchyma. Combining these systematic analyses, we not only provide a comprehensive insight into how different signalling networks are modulated in vivo during the different stages of $C$. neoformans infection, but also unravelled a large number of TFs and kinases involved in the brain infection of the pathogen. Most importantly, we demonstrate that the homeobox TF Hobl is the master regulator for a number of brain-infection-related genes in the C. neoformans H99 strain, but not in the Cryptococcus deuterogattii R265 strain that does not target the brain during infection.

\section{Results}

Brain infectivity assay with $C$. neoformans kinase and TF mutants. We previously performed STM-based murine lung infectivity assays with the kinase and TF mutant libraries (264 strains representing 129 kinases and 322 strains representing
155 TFs) using the lungs recovered after 14 days post infection (dpi) from intranasally infected mice and identified 58 and 40 lung infectivity-related kinases ${ }^{15}$ and $\mathrm{TFs}^{16}$, respectively. These may represent the signalling components required for the initial stage of cryptococcal infection. To identify the signalling components required for the brain infection, which represents the late stage of cryptococcal infection, the STM scores of TF and kinase mutants recovered from the brain tissues of the same infected mice were measured (Supplementary Fig. 1). However, we were often unable to recover a sufficient amount of $C$. neoformans cells from the brain tissues of some mice for STM analysis. In addition, as the intranasally infected $C$. neoformans mutants should pass through the lungs first, those reaching the brain were no longer equally distributed unlike pooled input mutants, rendering direct comparison of the lung and brain-STM scores difficult. Therefore, we performed another round of STM-based murine infectivity assay for the TF and kinase mutant libraries using the brain recovered from intravenously infected mice after $7 \mathrm{dpi}$ (see the strategy of Fig. 1a) and compared the brain-STM score with the previous lung-STM score for each mutant (Fig. 1b, c; Supplementary Fig. 2).

Regarding kinases, the 34 kinases that were found to be required for both the lung and brain infections were defined as core-virulence kinases. Some of them were signalling components of previously characterised signalling pathways in C. neoformans: Pka1 in the cAMP signalling pathway ${ }^{17,18}$, Ssk2 and Hog1 in the high osmolarity glycerol response (HOG) pathway ${ }^{19,20}$, Bck1 and Mpk1 in the cell wall integrity MAPK pathway ${ }^{21,22}$, Ire1 in the unfolded protein response (UPR) pathway ${ }^{23}, \mathrm{Vps} 15$ in the vacuole-trafficking pathway ${ }^{15}$, Snf1 and Gal83 in the carbon utilisation pathway ${ }^{15}$, Bud32 in the KEOPS/EKC complex, Ypk1, Gsk3, and Ipk1 in the the TOR (Target of Rapamycin) pathway ${ }^{15}$. Except for these known proteins, the core virulence-related kinases also include Irk 2 and Irk5, whose regulatory mechanisms are not evident. Irk2 and Irk5 belong to the families of APH phosphotransferases, diacylglycerol kinase-like kinase and AGC/ YANK protein kinase, respectively. Deletion of IRK5 significantly reduces melanin production, but enhances capsule production ${ }^{15}$, indicating that defective melanin formation may be attributable to the role of Irk5 in virulence. However, deletion of IRK2 did not result in evident in vitro phenotypic alteration. In contrast to the 34 core-virulence kinases, 23 and 12 kinases were required only for the lung or brain infection, respectively (Fig. 1b; STM cutoff $>2.0$ or $<-2.0 ; P<0.05$ ).

In the case of TFs, a total of 9 TFs were found to be required for both lung and brain infections and here defined as corevirulence TFs. These include Sre1 and Hob1 in the sterol biosynthesis pathway ${ }^{16}$, Hxl1 in the UPR pathway ${ }^{23}$, and Gat201 and Nrg1 in the capsule biosynthetic pathway ${ }^{24,25}$. Besides these known TFs, Pdr802, Fzc1, Fzc9 and Fzc31, which all contain the fungal specific $\mathrm{Zn}_{2} \mathrm{Cys}_{6}$ DNA binding domain, were also identified as core-virulence TFs. Notably, in vivo functions of these four TFs attributed to the pathogenicity of $C$. neoformans were not evident based on their in vitro mutant phenotypic traits ${ }^{16}$. Deletion of $F Z C 1$ reduced the growth at $39^{\circ} \mathrm{C}$ (but not at $37^{\circ} \mathrm{C}$ ) and mating capacity but increased capsule and melanin production. Deletion of FZC9 reduced mating and resistance to hydrogen peroxide. Deletion of FZC31 reduced the growth under high temperature and oxidative stresses and mating capacity but increased melanin production. In contrast to the 9 core-virulence kinases, 23 and 10 TFs were required only for the lung or brain infection, respectively (Fig. 1c; STM cutoff $>2.0$ or $<-2.0$; $P<0.05)$.

Overall, deletion of kinase genes resulted in more dramatic changes in both the lung and brain-STM scores than deletion of TFs did, probably because kinases generally function upstream of 
a
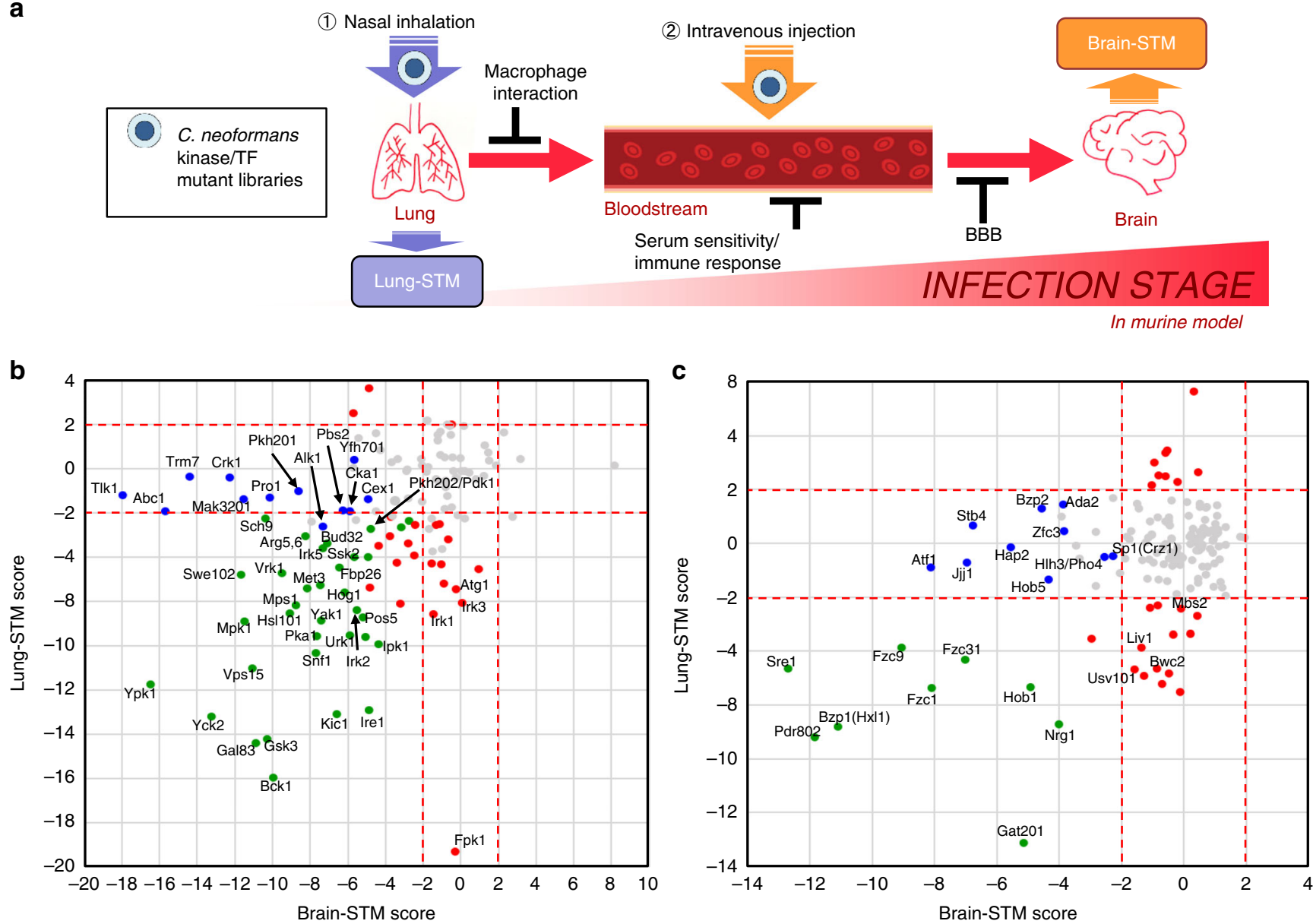

Fig. 1 Dual signature-tagged mutagenesis (STM) analyses of kinase and TF deletion libraries of $\boldsymbol{C}$. neoformans. (a) A schematic diagram for comparison of lung (via intranasal infection) and brain (via intravenous infection) STM analysis using C. neoformans TF and kinase mutant libraries ${ }^{15,16}$. (b-c) Lung-STM scores for each mutant were obtained from the previous reports using a intranasally infected murine model $(A / J$ mice, $n=3)$. Brain-STM scores were obtained in this study using the A/J mouse model intravenously infected with the same set of TF and kinase mutant libraries (the whole data were shown in Supplementary Fig. 2). Dual STM scores of (b) 129 kinases and (c) 155 TFs were represented by brain-STM score (X-axis) and lung-STM score (Y-axis). Each spot was an average STM score of two-independent TF or kinase mutant strains, each of which was obtained from three mice $(n=3)$. We used the colour code for kinase/TF mutants that exceeded STM cutoff $[>2.0$ (high) or $<-2.0$ (low) and had statistically significant $P$ values ( $<0.05$ ) by one-way ANOVA analysis with Bonferroni's multiple comparison test. Green dots indicate the core-virulence kinase/TF mutants that show low/high STM scores in both lung- and brain infections. Blue dots indicate the kinase/TF mutants that show low/high STM scores only in brain-infection. Red dots indicate the kinase/TF mutants that show low/high STM only in lung infection.

TFs in signalling pathways. These results clearly indicate that redundant and distinct signalling components are involved in different cryptococcal infection stages.

In vivo transcription profiles of kinases and TFs during infection. To further gain insight into the in vivo regulatory mechanisms of pathogenicity-related kinases and TFs at different C. neoformans infection stages, we performed NanoString nCounter-based in vivo transcription analysis of 180 TFs and 183 kinases, along with 58 known virulence-related genes (See Supplementary Data 1). This platform has been effectively used to quantitatively measure in vivo gene expression of target genes of a pathogen during host infection ${ }^{26}$. For this analysis, mice were intranasally infected with the C. neoformans H99 strain and infected lung, brain, kidney, and spleen tissues were recovered after 3 (early stage), 7 (middle stage), 14, and 21 dpi (late stages) (Fig. 2a). Total murine and cryptococcal RNAs were isolated from each infected tissue and used for NanoString assays to quantitate pathogen-specific mRNA transcripts using designed probes by normalisation with average expression levels of eight housekeeping genes (Supplementary Data 1). In vivo expression levels of each target gene at different tissues and days of infection were compared to their in vitro expression levels under basal growth conditions [yeast extract-peptone-dextrose (YPD) medium at $30^{\circ} \mathrm{C}$ ].

We first checked how 58 known virulence-related genes are differentially regulated during infection. Among these, genes involved in metal ion sensing and uptake, such as CIG1 and CFO1, were highly upregulated at different tissues at all infection stages (Fig. 2b), further supporting that Cig1 and Cfol are essential for virulence of C. neoformans ${ }^{27,28}$. The copper regulon genes such as CnMT1/2 (metallothioneins) and CTR4 (copper transporter) were also highly upregulated at all infected tissues from the early to the late infection stages. In addition, genes involved in the production of two major virulence factors, melanin (LAC1) and capsule (CAP10, CAP59, CAP60, CAP64), were differentially regulated during infection. Notably, in vivo expression levels of LAC1 increased during 3 14 dpi and decreased at $21 \mathrm{dpi}$, implying that $L A C 1$ induction is associated with the survival and adaptation of $C$. neoformans in the host. 
a
Inhalation infection

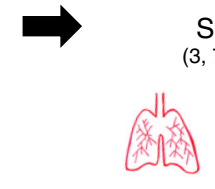
Sampling infected tissues $(3,7,14$, and 21 days post infection)
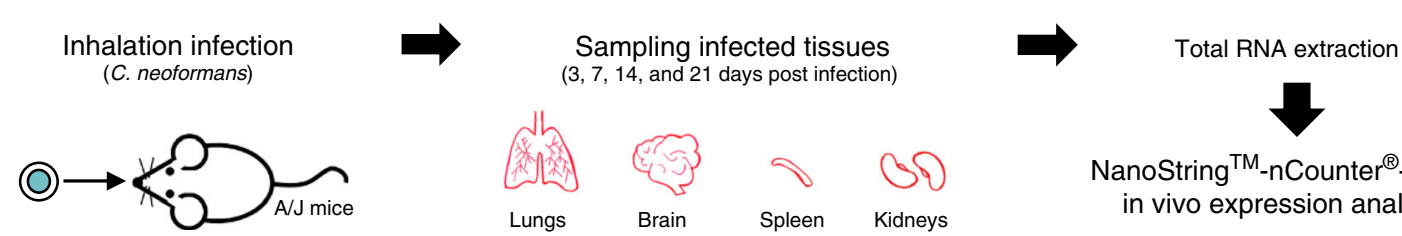
NanoString ${ }^{\mathrm{TM}}$-nCounter ${ }^{\circledR}$-based in vivo expression analysis
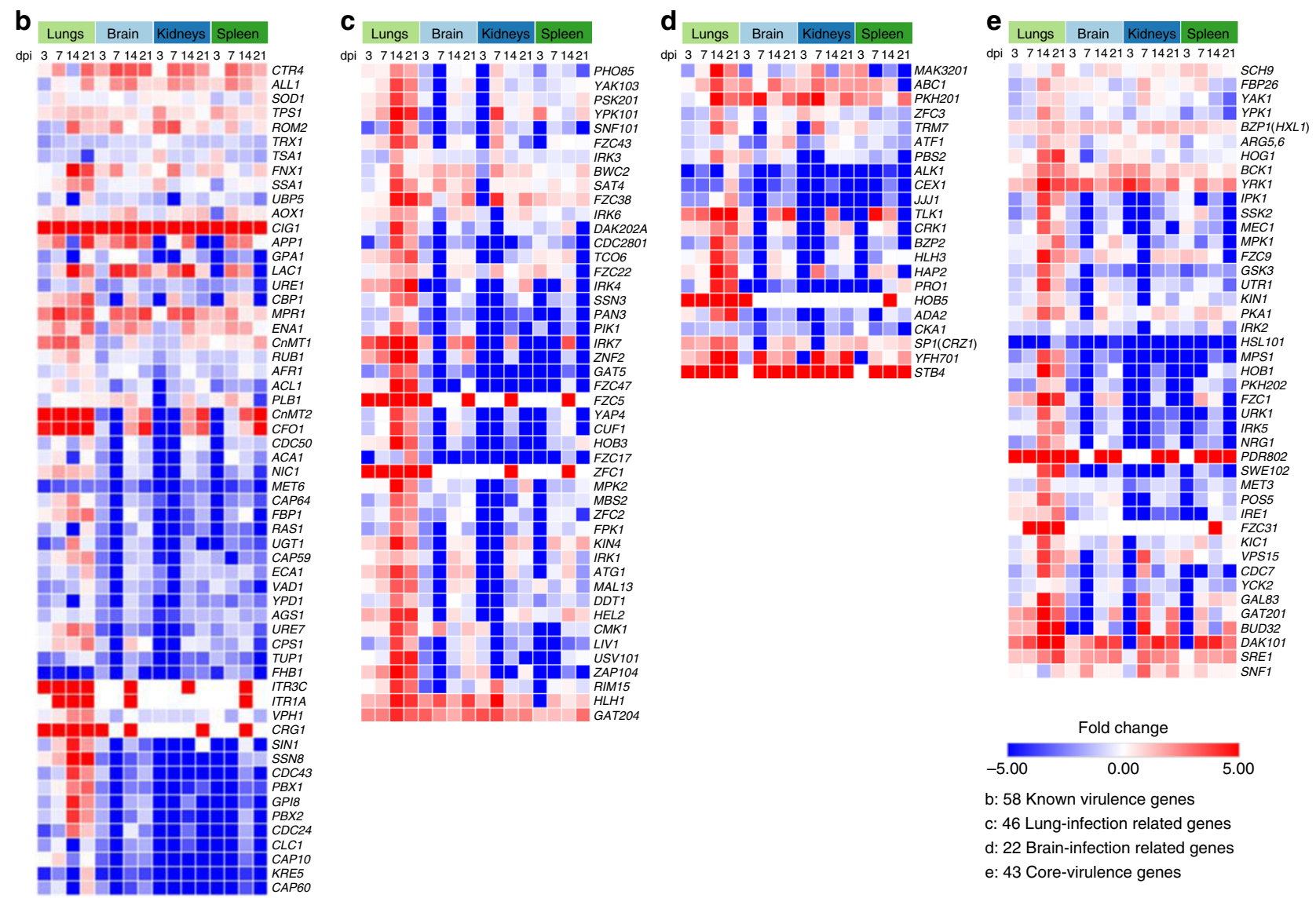

Fig. 2 In vivo expression profiling of $\boldsymbol{C}$. neoformans transcription factor and kinase genes in comparison with known cryptococcal virulence genes. (a) Graphical abstract of NanoString ${ }^{\top M_{-}}{ }_{-}$Counter $^{\circledR}$ based in vivo gene expression analysis in a murine model of systemic cryptococcosis through intranasal instillation. Total RNA was extracted from the infected lung, spleen, kidney, and brain tissues recovered after 3, 7, 14, and 21 dpi. Three mice per cohort were used (total 12 mice). Gene-specific nCounter codesets were designed for 58 published virulence-related genes, 183 kinases, 180 TFs, and 8 housekeeping genes for expression normalisation (Supplementary Data 1 and Supplementary Fig. 3). (b-e) Fold-change heatmap of in vivo gene expression of (b) 58 published (known) virulence-related genes, (c) 42 lung-infection-related genes, (d) 22 brain-infection-related genes, and (e) 43 corevirulence genes.

Unexpectedly, in vivo expression of CAP10, CAP59, CAP60, and CAP64 was generally downregulated in all infected tissues except the lungs where they were upregulated by about 2 fold between 7 to 21 dpi (Fig. 2b).

The in vivo transcription profiling analysis revealed the following expression patterns of 183 kinase and 180 TF genes during the whole infection process. Reflecting that the lungs are the initial infection sites for C. neoformans, expression of a large number of kinases and TF genes were induced in the lungs, particularly after $14 \mathrm{dpi}$ (Supplementary Fig. 3). Notably, we found that no genes were specifically expressed only in the spleens, kidneys, or brains. Collectively, these results indicated that transcriptional modulation of pathogenicity-related kinases and TFs appeared to be initiated at the lung infection stage of cryptococcal infection.

Kinases and TFs required for adhering and crossing the BBB. We further focused on the 12 kinase and $10 \mathrm{TF}$ mutants that showed significantly reduced brain-STM score (cutoff $<-2.0 ; P<$ 0.05 ), but not in the lung (Fig. 1b, $c$ and Supplementary Data 2). The change in the brain-STM score could occur during haematogenous dissemination. Therefore, we monitored the serum sensitivity of these TF and kinase mutants in the presence of $50 \%$ foetal bovine serum (FBS) in YPD medium. Surprisingly, only the pho4s mutant (also known as hlh $3 \Delta$ ) showed marked serumspecific growth defect (Supplementary Fig. 4), which is in agreement with the previously reported data ${ }^{29}$. Therefore, the low brain-STM score of the pho $4 \Delta$ mutant, at least partly, resulted from its serum sensitivity.

For the remaining low brain-STM TF and kinase mutants, we hypothesised that some of these mutants could have altered the ability to traverse the BBB. Previously, the in vitro BBB system, which consists of human brain microvascular endothelial cells (HBMECs) growing on a transwell membrane separating the top compartment (blood site) and bottom compartment (brain side), had been constructed by $\mathrm{Vu}$ et al. ${ }^{30}$ and successfully employed to 
discover BBB-traversal factors, including $\mathrm{Mpr}^{31}$. In this study, we similarly established the transwell-based in vitro BBB system and tested the capability of the selected brain-infection-related TF and kinase mutants in traversing the BBB. To verify our in vitro $\mathrm{BBB}$ system, we first compared the BBB-traversal ability of $C$. neoformans (H99 strain) with non-pathogenic $S$. cerevisiae (S288C) and the mpr1s mutant (Supplementary Fig. 5) that was independently constructed in this study. After $24 \mathrm{~h}$ incubation, about $10 \%$ of the wild-type (WT) C. neoformans were able to traverse the $\mathrm{BBB}$ (Fig. 3a). In contrast, $S$. cerevisiae did not traverse the $\mathrm{BBB}$ at all and the $C$. neoformans mpr $1 \Delta$ mutant was highly defective in BBB traversal (less than 2\%) (Fig. 3a). Similar to the previous finding ${ }^{32}$, we did not find any significant changes in trans-endothelial electrical resistance (TEER) (Fig. 3a), further verifying that the tight junction joining HBMECs was not affected during the $\mathrm{BBB}$ traversal of $C$. neoformans cells.

We next assessed the 12 kinase and 10 TF mutants, which showed low STM score in the brain, but not in the lung, for their capability to traverse the BBB. Among these, we excluded the $c k a 1 \Delta$, pro $1 \Delta$, and $b z p 2 \Delta$ mutants in this analysis, as they exhibit significantly retarded growth at $37^{\circ} \mathrm{C}^{15,16}$. We found that a total of 5 kinases (Cex1, Alk1, Pbs2, Yfh701, and Pkh201) and 4 TFs (Ada2, Hap2, Pho4, and Jjj1), whose deletion all resulted in low brain-STM scores, were required for the BBB traversal (Fig. 3b). As the first step for the BBB traversal of $C$. neoformans is its adhesion to the surface of the HBMECs, we further examined the capability of the BBB-crossing defective 5 kinase and $4 \mathrm{TF}$ mutants to adhere to the monolayer of HBMECs. Among these,

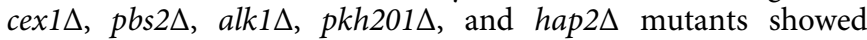
reduced adhesion to HBMECs (Fig. 3c), indicating that host cell adhesion is an important pre-requisite for efficient BBB crossing of C. neoformans (summarised in Supplementary Data 2).

We also examined the BBB crossing ability of core-virulence 34 kinase and 9 TF mutants except those defective in growth at $37^{\circ} \mathrm{C}$ $(y c k 2 \Delta, \arg 5,6 \Delta, f b p 26 \Delta, m p s 1 \Delta, \operatorname{mec} 1 \Delta, s w e 102 \Delta, c d c 7 \Delta, g s k 3 \Delta$,

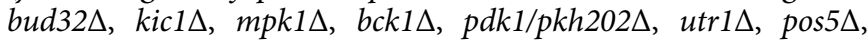
$i p k 1 \Delta$, ire $1 \Delta, v p s 15 \Delta, y p k 1 \Delta, n r g 1 \Delta$, and $h x l 1 \Delta)$. Among the remaining 15 kinase and 7 TF candidates, hog $1 \Delta, s s k 2 \Delta$, pka1 , and gat $201 \Delta$ mutants were as efficient in BBB crossing as WT strain, suggesting that Hog1 MAPK, cAMP/PKA, and Gat201dependent pathways are not involved in BBB crossing (Fig. 3d). Interestingly, the finding that the Pbs2 MAPK kinase (MAPKK), but not its downstream Hog1 MAPK, was involved in BBB crossing indicated that $\mathrm{Pbs} 2$ may have downstream targets other than Hog1 for regulating $\mathrm{BBB}$ crossing. In contrast, Sre1 and Hob1 in the sterol biosynthesis pathway, Sch9 in the TOR pathway, Met3 in the sulphur assimilation pathway, Snf1 and Gal83 in the carbon utilisation pathway, and Vrk1 in ribosome biogenesis pathway appear to promote $\mathrm{BBB}$ crossing of $C$. neoformans (Fig. 3d). In addition to these kinases and TFs with known pathways, Hsl101, Irk2, Irk5, Urk1, Fzc1, Fzc9 and Pdr802 in undefined pathways were also found to be critical for BBB

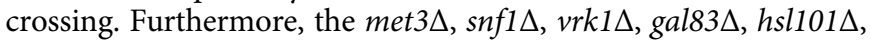
irk $2 \Delta$, sre $1 \Delta$, pdr802 $\Delta, f z c 9 \Delta$, hob $1 \Delta$, and $f z c 1 \Delta$ mutants, but not irk $5 \Delta$, sch $9 \Delta$, and urk $1 \Delta$ mutants, showed reduced adhesion to the monolayer of HBMECs, further supporting that host cell adhesion is a critical pre-requisite for efficient BBB crossing of $C$. neoformans (Fig. 3e). Collectively, these data suggest that $C$. neoformans employs complex signalling networks involved in a variety of biological processes to cross the BBB (summarised in Supplementary Data 3).

Notably, however, the NanoString-based in vivo transcription profiling analysis revealed that these brain infectivity-related kinases and TFs were not specifically modulated only in the brain. In fact, most of these genes were upregulated in the lungs at the later stage of infection (14 and $21 \mathrm{dpi}$ ) (Fig. 3f). Particularly, in vivo expression of PDR802, SRE1, VRK1, PKH201, and YFH701 was strongly induced in all infected tissues tested during almost all infection stages (Fig. 3f). In contrast, regardless of the critical roles of Cex 1 and Met 3 in BBB crossing and adhesion to HBMECs, their in vivo expression levels were not strongly induced at all infection stages and in all tissues (Fig. 3f).

Kinases and TFs required for survival in the brain parenchyma. A question remains for the TF and kinase mutants, which were normal in haematogenous dissemination and crossing the BBB, but still showed significant changes in brain-STM score. We hypothesised that some of these mutants may lack an ability to survive in the brain parenchyma even after crossing the BBB. To address this question, we directly monitored the capability of the low brain-STM TF and kinase mutants to proliferate in the brain parenchyma. For this purpose, we established an intracerebroventricular (ICV) method of infection of the mouse brain with C. neoformans by bypassing the BBB (Fig. 4a), which allowed us to infect the brain parenchyma consistently and with an equal number of cryptococcal cells. Once we infected a group of mice with the low brain-STM TF and kinase mutants by ICV injection, we recovered mutants from the infected brain after $6 \mathrm{dpi}$ and assessed the STM score by qPCR (here abbreviated as ICV-STM score). We found that 6 strains with deleted kinases (TLK1, TRM7, CRK1, MAK3201, PKH201, or ALK1) and 4 with deleted TFs (ADA2, BZP2, ZFC3, or HAP2) displayed significantly reduced ICV-STM score (Fig. 4b,c). Among these, 2 kinases (Pkh201 and Alk1) and 2 TFs (Ada2 and Hap2) were required for both $\mathrm{BBB}$ crossing and survival in the brain parenchyma. Notably, the kinases and TFs that were required for BBB crossing (Cex1, Pbs2, Yfh701, Pho4, and Jjj1) were dispensable for proliferation in the brain parenchyma (Fig. 4b, c). In contrast, 4 kinases (Tlk1, Trm7, Crk1, and Mak3201) and a single TF (Zfc3) were uniquely involved in proliferation in the brain parenchyma, but not in BBB crossing (Fig. 4b, c).

We also monitored ICV-STM score for 30 and 9 core-virulence kinase and TF mutants. As expected, most kinase and TF mutants that were defective in growth at $37^{\circ} \mathrm{C}(y c k 2 \Delta, \arg 5,6 \Delta, m p k 1 \Delta$, mps $1 \Delta$, kic1 1 , bud $32 \Delta$, bck1 $1 \Delta$, utr $1 \Delta$, fbp $26 \Delta$, pos $5 \Delta$, mec1 1 , $i p k 1 \Delta$, swe 102 , and $n r g 1 \Delta$ mutants) also showed reduced ICVSTM score (Fig. 4d, e). Among the core-virulence kinases and TFs that were required for BBB crossing, Irk2, Vrk1, Pdr802, Sre1, and Hob1 were also required for proliferation in the brain parenchyma (Fig. 4d, e). Interestingly, the hog $1 \Delta$ mutant showed low ICV-STM score (Fig. 4d), indicating that Hog1 is not required for $\mathrm{BBB}$ crossing, but required for proliferation in the brain parenchyma. In contrast, Sch9, Irk5, Fzc1, and Fzc9 were dispensable for survival in the brain parenchyma (Fig. 4d, e). In addition, we found that Snf1, Gal83, Kin1, Urk1, Hsl101, Yak1, Fbp26, Pos5, and Swe102 were involved in the proliferation in the brain parenchyma. Collectively, our data clearly indicated that $C$. neoformans employs redundant and distinct sets of signalling pathways to cross the $\mathrm{BBB}$ and proliferate in the brain parenchyma.

Hob1 is a master regulator of brain infectivity in C. neoformans. We next aimed to address how regulatory networks of known brain-infection factors, such as Ure1, Cps1, Itr1a, Itr3c, Mpr1, Plb1, Fnx1, and Rub1, are connected to brain-infectionrelated kinases and TFs identified in this study. To this end, first we examined whether the known brain-infection factors are transcriptionally regulated by in vitro host-mimic conditions (HMC): a tissue culture medium (RPMI) supplemented with $10 \%$ FBS at $37^{\circ} \mathrm{C}$ under $5 \% \mathrm{CO}_{2}$. We found that expression of ITR1a, $I T R 3 c$, and MPR1 was strongly induced ( 3-4 folds) by HMC 
a

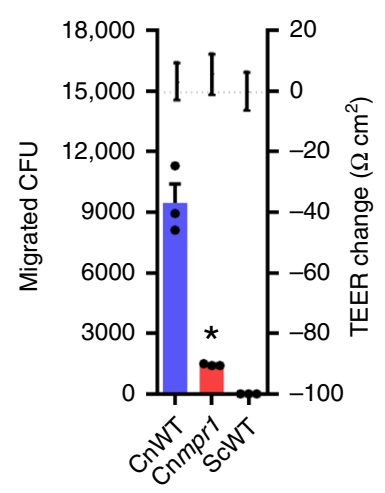

b

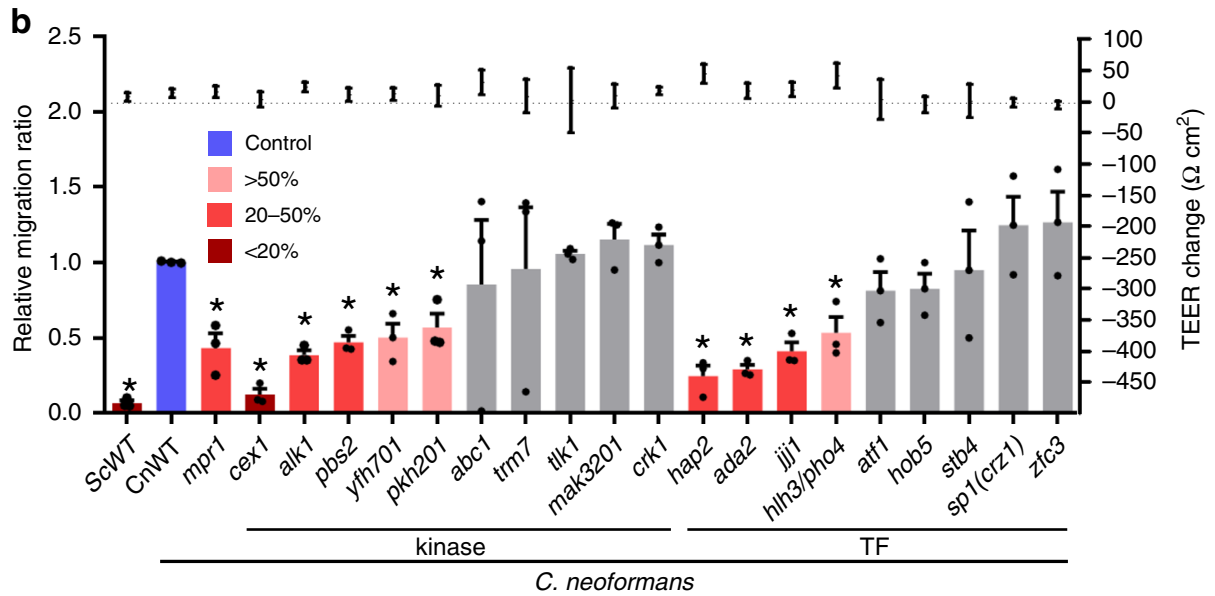

C

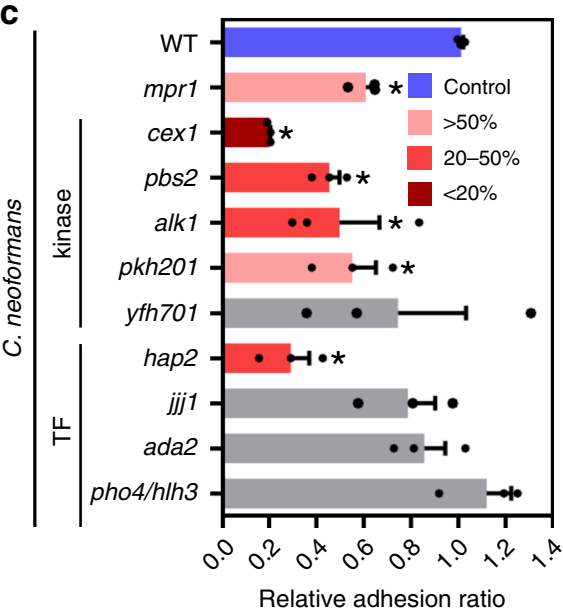

e

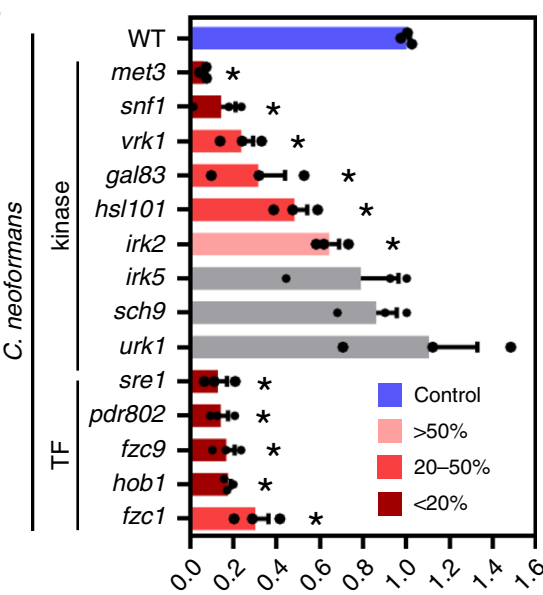

d

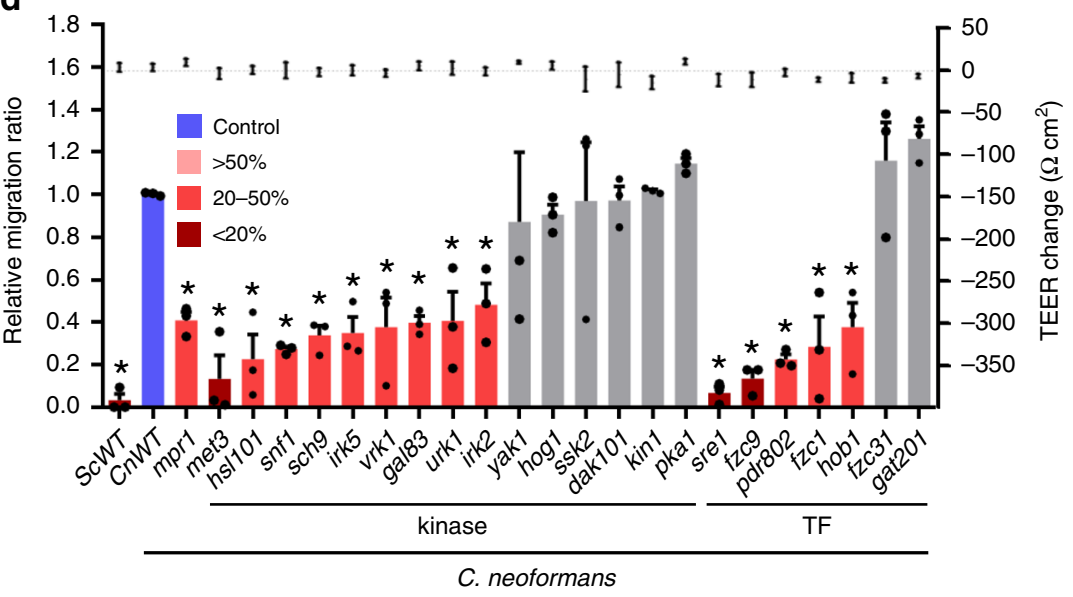

$\mathbf{f}$

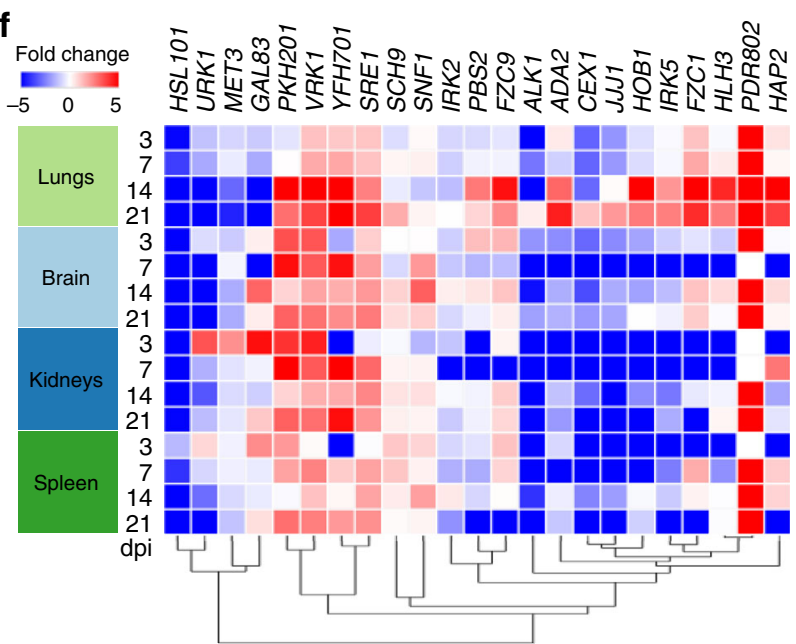

Fig. 3 In vitro BBB transmigration and adhesion assays for $C$. neoformans transcription factor and kinase mutants having low brain STM scores. BBB transmigration assays with hCMEC/D3-coated Transwell systems ( $\mathbf{a}, \mathbf{b}, \mathbf{d})$ and hCMEC/D3 cell adhesion assays (c, e). a, b, $\mathbf{d}$ Plates with inoculum of $10^{5}$ yeast cells were incubated at $37^{\circ} \mathrm{C}$ in $\mathrm{CO}_{2}$ incubator for $24 \mathrm{~h}$, and the number of yeast cells passing through the hCMEC/D3-coated Transwell was measured by CFU. S. cerevisiae WT (SCWT, S288C) and C. neoformans mpr1 mutant (Cnmpr1) were used as negative controls. a Left and right $Y$ axes indicate the migrated CFU and the trans-endothelial electrical resistance (TEER) value, respectively. $\mathbf{b}, \mathbf{c}$ The results of BBB transmigration (b) and adhesion assays (c) of brain-infection-related kinases and TFs discovered by brain-STM analysis. Left and right $Y$ axes indicate the relative migration ratio and the TEER value, respectively. $\mathbf{d}$, e The results of BBB transmigration (d) and cell adhesion assay (e) of core-virulence kinases and TFs discovered by dual STM analyses. Each mutant was analysed by more than three biologically independent experiments with three technical replicates. List of strains used in this Figure is described in Supplementary Data 4. a-e Data are presented as mean values \pm standard error of the mean (SEM). Statistical significance of difference between CnWT (H99 strain) and each mutant $\left({ }^{*}, P<0.05\right)$ was calculated by two-tailed Student's $t$ test (unpaired, $n=3$ ). $\mathbf{f}$ NanoString heatmap of BBB-crossing-related kinases and TFs was clustered by using Morpheus. 
a
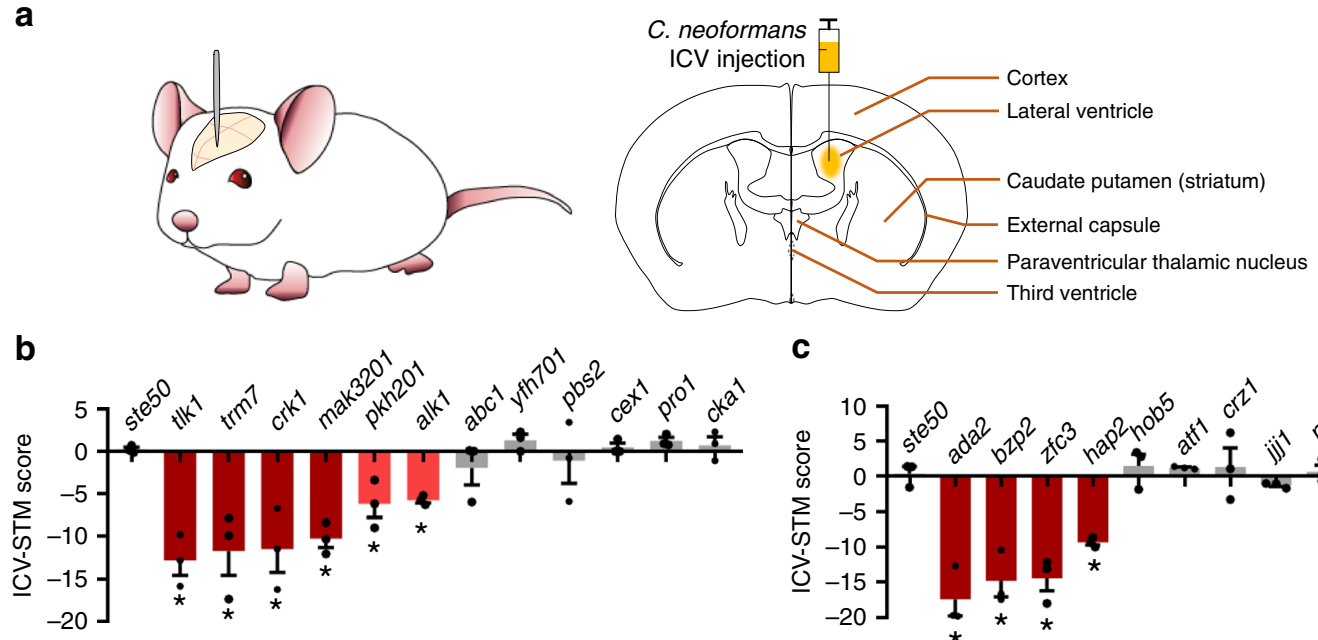

C

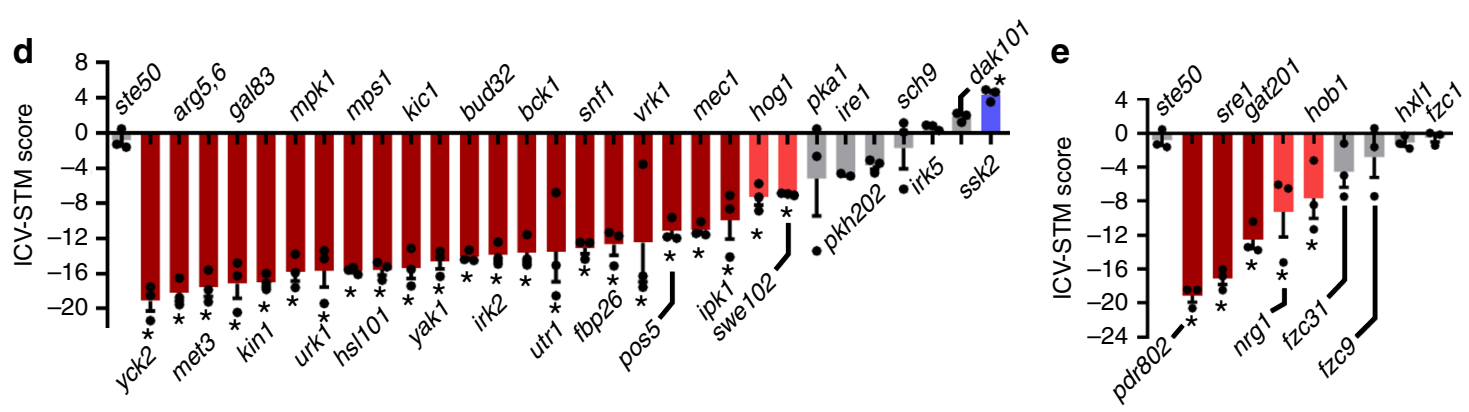

Fig. 4 Identification of genes required for survival of $C$. neoformans in the brain parenchyma through intracerebroventricular (ICV)-STM analysis. (a) Graphical abstract of ICV injection for directly infecting the brain parenchyma with C. neoformans. (b-e) ICV-STM score of brain-infection-related (b) kinases, (c) TFs, core-virulence (d) kinases, and (e) TFs. Three mice (A/J) were infected with each pooled mutant group (Supplementary Data 4), sacrificed after $7 \mathrm{dpi}$, and infected brain tissues were harvested for recovering cryptococcal cells. The ICV-STM score was calculated by quantitative PCR of genomic DNA isolated from input and output cryptococcal mutants as described in Methods. The ste50 $\Delta$ mutant was used as a virulent control strain. Red and blue marks (with *) indicate mutants whose STM scores are statistically different $(P<0.05, n=3)$ from the ste50 $\Delta$ STM score by one-way ANOVA analysis with Bonferroni's multiple comparison test. Data are presented as mean values \pm SEM.

(Fig. 5a), further indicating that inositol transporters and metalloprotease are critical for $\mathrm{BBB}$ crossing. We also monitored whether expression of any BBB-crossing-related TFs that were discovered in this study were also induced by HMC. Except for PHO4, none of brain-infection-related TFs described in Fig. 3b (ADA2, HAP2, and JJJ1) were induced by HMC (Fig. 5b). We then paid attention to some of the core-virulence TFs that were required for both lung and brain infections: Fzc1, Fzc9, Fzc31, Pdr802, Hob1, Sre1, Hxl1, Nrg1, and Gat201 (Fig. 1c). Expression of FZC31, PDR802, HOB1, SRE1, HXL1, and GAT201 was induced by HMC, whereas expression of FZC9 and NRG1 was rather repressed (Fig. 5c).

Among these, we further focused on Pdr802, Hob1, and Sre1, because their expression was induced by HMC and they were required for both $\mathrm{BBB}$ crossing and proliferation in the brain parenchyma. Moreover, the in vivo expression of PDR802 and $S R E 1$ was strongly induced at all infected tissues throughout the whole infection stages, although $H O B 1$ expression was induced at the lungs only after $14 \mathrm{dpi}$ (Fig. $5 \mathrm{~d}$ ). Therefore, we hypothesised that these TFs could be potential candidates for regulating HMCinducible genes. To test this hypothesis, we assessed the expression levels of HMC-inducible genes in sre $1 \Delta$, hob $1 \Delta$, and pdr802 $\Delta$ mutants in comparison with those of the WT strain. Surprisingly, HMC-mediated induction of ITR $1 a$, ITR $3 c$, and $M P R 1$ was markedly reduced in the hob1 $\triangle$ mutant, but not in the

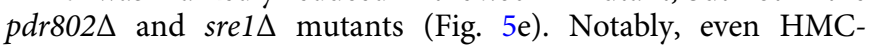
induced PDR802 and SRE1, along with FZC31, were significantly reduced in the hob1 $\Delta$ mutant (Fig. 5e). In contrast, GAT201 was induced normally by HMC in the hob1 $\triangle$ mutant, suggesting that GAT201 expression may be controlled by other TFs. The HMCmediated induction of FZC31 was also regulated by Sre1 as well as Hob1 (Fig. 5e). Collectively, these data indicate that Hob1 is a key regulator of induction of many brain-infection-related genes.

Hob1 is dispensable for the pathogenicity of $C$. deuterogattii. The critical role of Hobl in the regulation of brain-infectionrelated genes in C. neoformans prompted us to question its role in its pathogenic Cryptococcus sibling species $C$. deuterogattii, which is known to mainly target the lungs, but not the brain during infection $^{33,34}$. Among various $C$. deuterogattii strains, here we focused on the R265 strain, which belongs to the VGIIa clade and is most widely used for pathobiological study of C. gattii species complex ${ }^{35,36}$. The R265 strain contains a single conserved Hob1 orthologue in its genome (score: 1015, e-value: 0.0 ). Therefore, we constructed the hob1 $\Delta$ mutants in the R265 strain background (Supplementary Fig. 6) and compared their phenotypes with those of H99 hob1 $\Delta$ mutants. The H99 hob1 $\Delta$ mutants exhibited thermosensitivity, defects in melanin production, increased sensitivity to osmotic shock, oxidative stress, genotoxic stress, cell membrane/wall stress, and defective virulence ${ }^{16}$ (Fig. 6a and Supplementary Fig. 8). Notably, the hob1s mutants displayed high resistance to fluconazole, but increased susceptibility to amphotericin B, suggesting the role of Hob1 in ergosterol biosynthesis. Re-integration of the WT copy of $H O B 1$ into its native 


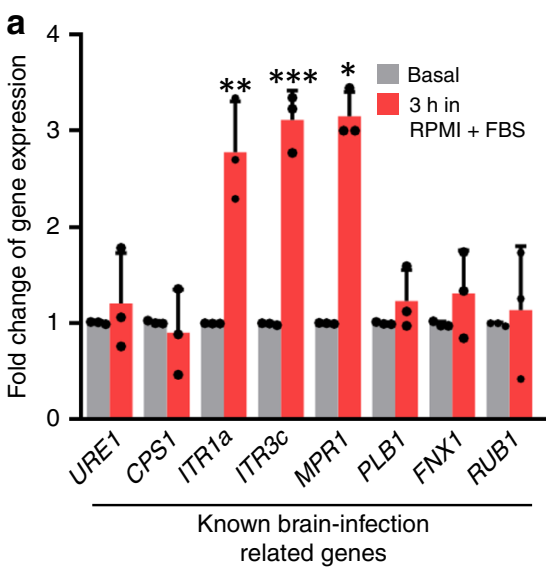

d

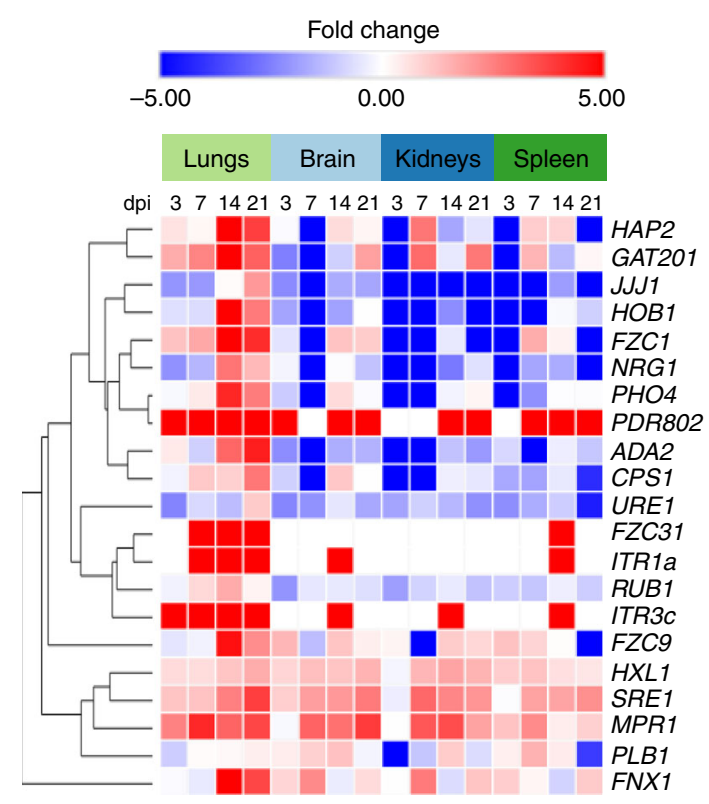

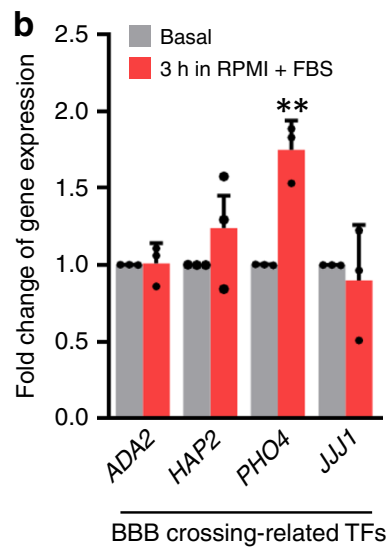

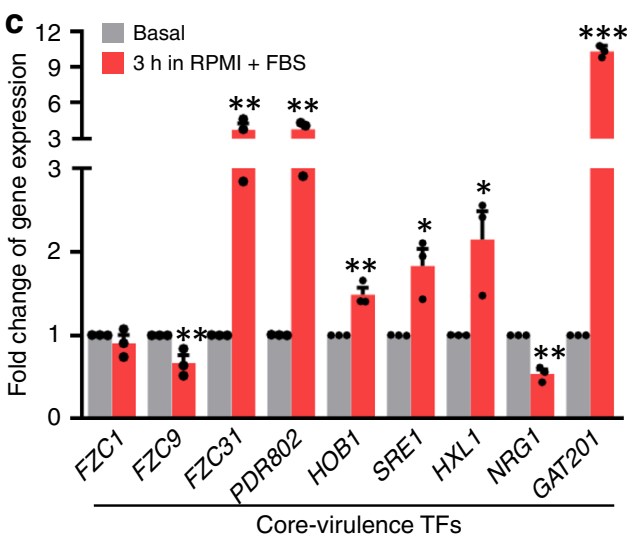

e
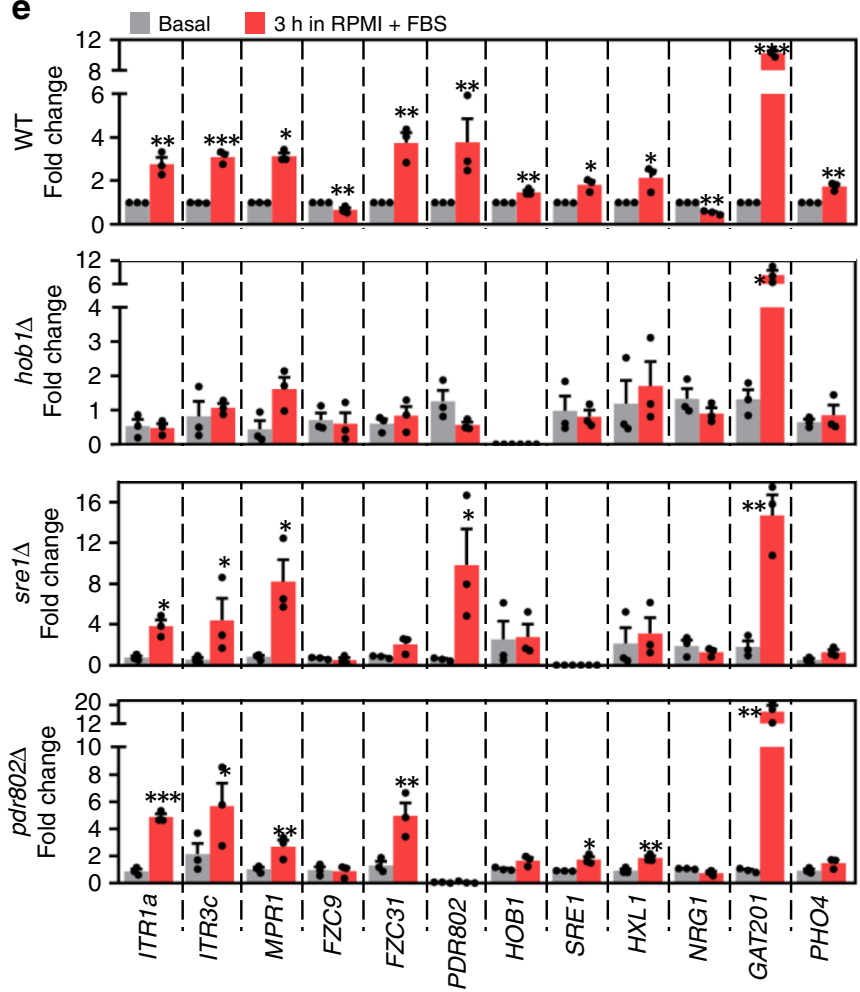

Fig. 5 The homeobox transcription factor Hob1 as a master regulator of brain-infection-related genes in $\mathbf{C}$. neoformans. a-c, e Host-mimic condition (HMC) mediated induction of known brain-infection-related genes (a), BBB crossing-related TFs (b), and core-virulence TFs (c). Expression of each gene

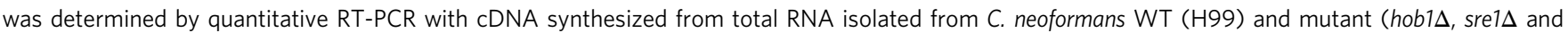
pdr802 ) strains that were shifted from basal condition (YPD at $30^{\circ} \mathrm{C}$; control samples; grey bars) to $\mathrm{HMC}$ (RPMI with $10 \%$ foetal bovine serum at $37{ }^{\circ} \mathrm{C}$ under $5 \% \mathrm{CO}_{2}$ ) and incubated for $3 \mathrm{~h}$ (red bars). Fold change of gene expression was calculated relative to the basal expression levels of each gene. Data from three independent experiments performed in duplicates are presented as mean values \pm SEM. Statistical significance of difference in gene expression levels between basal and HMC condition (unpaired, $n=3$ ) was determined by two-tailed Student's $t$ test $\left(^{\star}, P<0.05 ;{ }^{\star \star}, P<0.001 ;{ }^{\star \star \star}, P<0.0001\right.$ ). d NanoString heatmap of genes used in (a-c) was clustered by using Morpheus. Bold letters indicated the genes whose expression level was induced or reduced under HMC.

locus restored WT phenotypes in the hob1د mutants, verifying the function of Hob1 (Supplementary Fig. 10). Deletion of HOB1 in C. deneoformans XL280 strain background resulted in similar phenotypic changes, albeit to a lesser extent than those in H99 strain background (Fig. 6a). Strikingly, however, HOB1 deletion did not cause any significant phenotypic changes in R265 strain background (Fig. 6a and Supplementary Fig. 8). In contrast, deletion of SRE1, which is a major sterol-regulating TF in the SREB pathway, resulted in similar phenotypic changes, as shown by the highly increased susceptibility to fluconazole, of both H99 and R265 strains (Fig. 6a and Supplementary Fig. 8), suggesting that Sre1 plays conserved roles in both strains.

R265 Hob1 with a shorter N-terminal extension domain (558 amino acids) exhibited 91.9\% identity to H99 Hob1 (591 amino acids) (Supplementary Fig. 9a). Their homeobox domains, which are located at the N-terminus, have two amino acid changes (Supplementary Fig. 9a). To address whether such differences in Hob1 sequences are responsible for their distinct functions, we constructed hob1 $\triangle:: H O B 1^{\text {R265 }}$ allele-swapping strains, in which the R265 HOB1 allele was integrated into the native HOB1 locus 
a

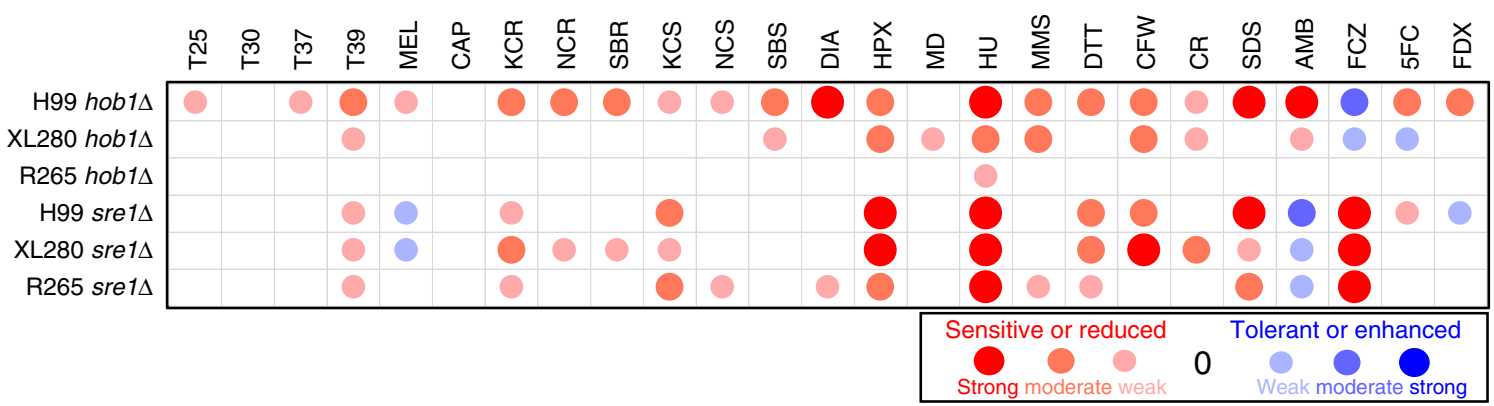

b
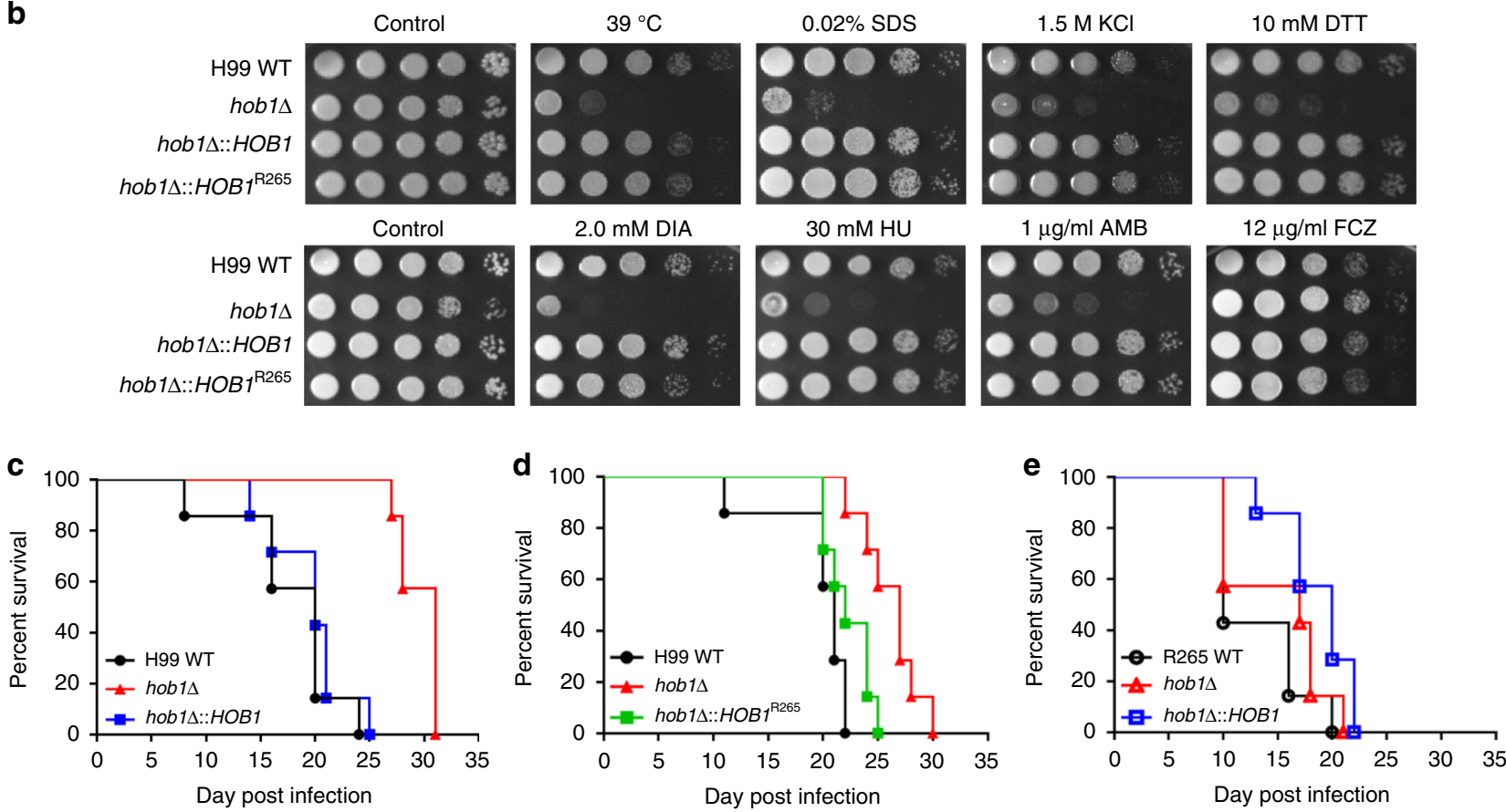

\begin{tabular}{|c|c|c|c|}
\hline$P$ value & WT & hob1s & $+\mathrm{HOB} 1$ \\
\hline & $\begin{array}{lll}- & \\
-1\end{array}$ & 0.0002 & 0.3780 \\
\hline hob1. & 0.0002 & - & 0.0002 \\
\hline
\end{tabular}

\begin{tabular}{|c|c|c|c|}
\hline & WT & hob1s & $+H O B 1^{\mathrm{R} 265}$ \\
\hline WT & - & 0.0007 & 0.0967 \\
\hline$b 1 \Delta$ & 0.0007 & - & 0.0002 \\
\hline
\end{tabular}

\begin{tabular}{|c|c|c|c|}
\hline & WT & hob1s & $+H O B 1$ \\
\hline WT & - & 0.3089 & 0.0914 \\
\hline$o b 1 \Delta$ & 0.3089 & - & 0.1216 \\
\hline
\end{tabular}

f

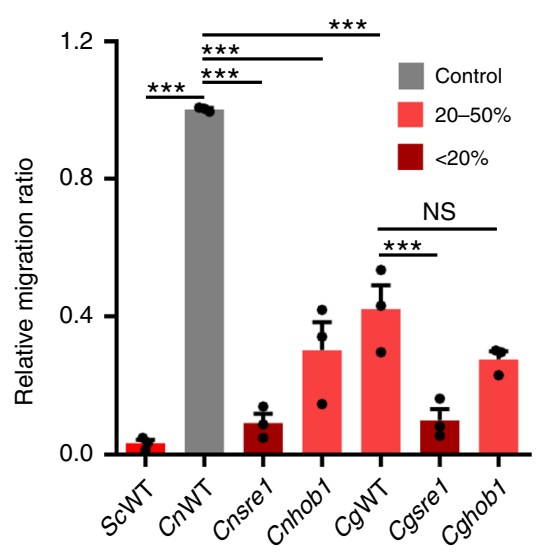

of the H99 hob1 $1 \Delta$ mutants (Fig. 6b and Supplementary Fig. 9b). Surprisingly, the R265 HOB1 allele restored all of WT phenotypic traits in the H99 hob1 $\Delta$ mutants (Fig. 6b), indicating that Hob1 orthologues can work interchangeably in both strains. Collectively, these results indicate that Hob1-downstream targets, not Hob1 per se, are functionally distinct between H99 and R265 strains.

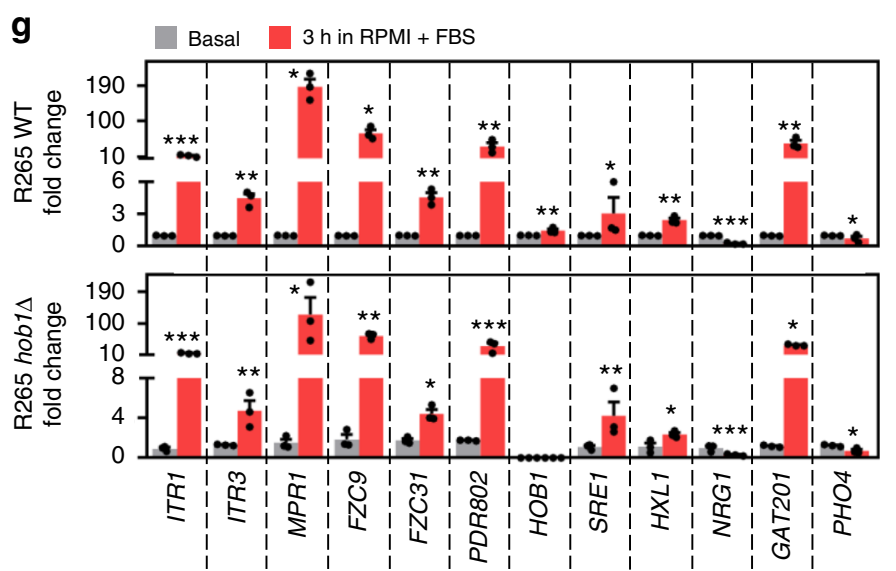

We next addressed the role of Hob1 in virulence of $C$. neoformans $\mathrm{H} 99$ and C. deuterogattii R265 strains using a murine (C57BL/6) intranasal instillation. As expected, the H99 hob1s mutant exhibited significantly attenuated virulence compared to WT and its complemented strain (hob1 $\Delta:: H O B 1$ ) (Supplementary Fig. 10a). In contrast, the R265 hob1 $1 \Delta$ mutant showed rather enhanced virulence compared to the WT and its complemented 


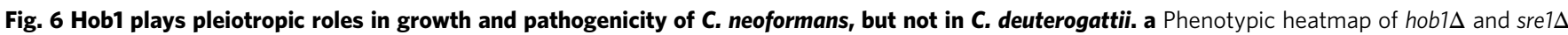
mutants constructed in C. neoformans H99, C. deneoformans XL280, and C. deuterogattii R265 strain backgrounds based on data presented in Supplementary Fig. 8, which shows one representative spot assay data of more than three independent experiments. Each abbreviation are defined as follows: T25, T30, T37, and T39: growth rates at $25^{\circ} \mathrm{C}, 30^{\circ} \mathrm{C}, 37^{\circ} \mathrm{C}$, and $39^{\circ} \mathrm{C}$; MEL: melanin production levels; CAP: capsule production levels; $\mathrm{KCR}$ : YPD + KCl; NCR: YPD + NaCl; SBR: YPD + sorbitol; KCS: YP + KCl; NCS: YP + NaCl; SBS: YP + sorbitol; DIA: diamide; HPX: hydrogen peroxide; MD: menadione; HU: hydroxyurea; MMS: methyl methanesulphonate; TM: tunicamycin; DTT: dithiothreitol; CFW: calcofluor white; CR: Congo red; SDS: sodium dodecyl sulphate; AMB: amphotericin B; FCZ: fluconazole; 5FC: 5-flucytosine; FDX: fludioxonil. b H99 WT, hob1 mutant, and HOB1 complementary and alleleswapping strains with $\mathrm{H} 99$ HOB1 or R265 HOB1 alleles were spotted onto YPD plates containing the indicated concentration of each chemical agent and photographed after 2-3 days. These spot assays were repeated more than three times, and one representative image was shown here. c-e Murine survival assay. A/J mice were infected by intranasal instillation with (c) C. neoformans WT (H99), hob14, and hob14::HOB1 strains constructed in H99 strain

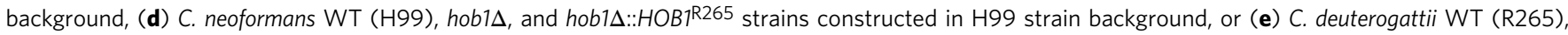

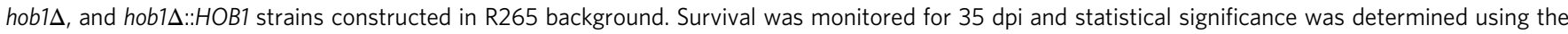
Mantel-Cox test by comparing a pair of indicated groups (7 mice per group, $n=7$ ). $\mathbf{f ~ B B B}$ crossing assay of hob1 $\Delta$ and sre1 $\Delta$ mutants in $\mathrm{C} n(\mathrm{H} 99)$ and $\mathrm{Cg}$ (R265). The Sc (S. cerevisiae S288C) strain was used as a negative control. Error bar indicates SEM for the three-independent experiments ( $n=3$ ), and statistics were calculated by one-way ANOVA with Bonferroni's correction. $\mathbf{g}$ HMC-mediated induction of brain-infection-related genes in C. deuterogattii WT (R265) and hob1s mutant as described in Fig. 5e. We performed three independent experiments $(n=3)$ with duplicates. Statistical significance of difference between basal and HMC condition was calculated by two-tailed Student's $t$ test (unpaired). Asterisks indicate the following range of $P$ values ( ${ }^{*}$, $\left.P<0.05 ;{ }^{\star \star}, P<0.001 ;{ }^{\star \star \star}, P<0.0001\right)$.

strains (Supplementary Fig. 10b). However, deletion of SRE1 abolished virulence in both H99 and R265 strains, whereas complementation with the SRE1 allele restored WT virulence in the sre1 $\Delta$ mutant (Supplementary Fig. 10). Next, we further confirmed the distinct roles of Hob1 in the virulence of H99 and R265 strains using the A/J mouse model. Similar to the results obtained using the C57BL/6 mouse model, the H99 hob1s mutant exhibited weakly but significantly attenuated virulence compared to WT $(P=0.0002)$ and its hob1 $\triangle:: H O B 1$ complemented strain $(P=0.0002)$ (Fig. 6c). Notably, the hob1 $\Delta:$ : $H O B 1^{\mathrm{R} 265}$ swapping strain exhibited wild-type levels of virulence $(P=0.0967)$ like the hob1 $\triangle:: H O B 1$ complemented strain (Fig. $6 \mathrm{~d})$, further indicating that Hob1 orthologues can work interchangeably in both strains during in vivo host infection. In contrast, the R265 hob1 $\Delta$ mutant showed WT virulence (Fig. 6e). These data indicate that Hob1 promotes the virulence of the C. neoformans H99 strain but not that of the C. deuterogattii R265 strain, while Srel plays a conserved role in the sibling Cryptococcus species complex.

To further examine the role of Hob1 in brain-infection of $C$. neoformans $\mathrm{H} 99$ and $C$. deuterogattii R265 strains, we monitored the ability of the hob1 $\Delta$ mutants in both strain backgrounds to traverse the $\mathrm{BBB}$. Reflecting the finding that C. deuterogattii does not target the brain during infection, the R265 strain did not cross the BBB as efficiently as the H99 strain, but traversed the BBB much more efficiently than $S$. cerevisiae (Fig. $6 \mathrm{f}$ ). Deletion of $\mathrm{HOB} 1$ markedly reduced the $\mathrm{BBB}$ crossing efficiency in the H99 strain, but not in the R265 strain (Fig. 6f). In contrast, sre1 $\Delta$ mutants exhibited reduced the $\mathrm{BBB}$ crossing ability in both strains, further confirming the conserved role of Sre1. Surprisingly, expression of ITR1 (ITR1a orthologue), ITR3 (ITR3c orthologue), MPR1, FZC9, FZC32, PDR802, and GAT201 was even more strongly induced by HMC in the R265 strain than in the H99 strain (Fig. 6g), indicating that these genes could not be responsible for discrepancy of the two Cryptococcus species. Notably, $\mathrm{PHO} 4$ expression was not induced by HMC in the R265 strain, unlike the H99 strain in which it was reduced (Fig. 6g). Notably, deletion of $H O B 1$ did not affect the HMC-mediated induction of ITR1, ITR3, MPR1, FZC9, FZC32, PDR802, SRE1, and GAT201, and HMC-mediated reduction of PHO4 (Fig. 6g) in the R265 strain. Collectively, the homeobox TF Hob1 play distinct roles in the pathogenicity of the C. neoformans H99 strain, but not in the pathogenicity of the C. deuterogattii R265 strain.

\section{Discussion}

In this study, we systematically dissected complex signalling pathways that govern each stage of the infection process of $C$. neoformans, which causes fungal pneumonia and fatal meningoencephalitis at the early and late infection stages, respectively. Here, we identified a large number of signalling components that are involved in initial lung infection, haematogenous dissemination, BBB adhesion and crossing, and proliferation in the brain parenchyma of $C$. neoformans, allowing us to understand how this basidiomycete fungal pathogen utilises distinct and redundant sets of signalling pathways during the whole infection process. The list of kinases and TFs and their biological functions required for the infection process are summarised in Fig. 7 and Supplementary Data 2 and 3, based on the known information available in C. neoformans or other fungi.

The following processes are considered to be core-virulence functions that are required for the whole infection process of $C$. neoformans: nutrient sensing/metabolism, cell growth and development, fatty acid and phospholipid biosynthesis, cell wall/ membrane integrity, inositol polyphosphate signalling, stress responses, tRNA modification, redox reaction and vacuole/protein sorting. Not surprisingly, based on our previous phenome database of the 111 infection-related TF/kinase genes $15,16,24 \%$ (22 kinases and $5 \mathrm{TFs}$ ) required for the growth at mammalian body temperature were essential for the whole spectrum of pathogenicity of C. neoformans (Fig. 7). Furthermore, 49\% (37 kinases and 17 TFs) and 36\% (30 kinases and 10 TFs) involved in capsule and melanin productions, respectively, were found to be core-virulence factors. Among the remaining genes, a majority of them were involved in stress response and adaptation. Therefore, our study further confirms that these virulence factors are critical for the whole infection processes of C. neoformans. As deletion of 12 kinases (Crk1, Dak202a, Fpk1, Irk1, Irk2, Irk3, Irk7, Mpk2, Psk201, Rim15, Snf101, and Trm7) and 16 TFs (Bwc2, Ddt1, Fzc17, Fzc22, Fzc38, Fzc5, Hel2, Jjj1, Mal13, Pdr802, Stb4, Yap4, $\mathrm{Zfc} 1, \mathrm{Zfc} 2, \mathrm{Zfc} 3$, and $\mathrm{Znf} 2$ ) did not result in any evident in vitro phenotypes, their essential in vivo functions need to be further elucidated in future.

Kinases and TFs, whose deletion resulted in reduced lung-STM scores but normal brain-STM scores, could play specific roles in lung infection (Fig. 7). Among these, the $f p k 1 \Delta$ mutant exhibits the lowest lung-STM score. However, deletion of FPK1 does not result in any phenotypic changes, although its overexpression suppresses defective growth, stress resistance, and cell wall/ membrane integrity of the $y p k 1 \Delta$ mutant, suggesting that Fpk1 
Core-virulence kinases and TFs for both lung and brain infections

Vrk1, Irk2, Gal83, Snf1, Kin1, Urk1, Hsl101, Yak1, Ssk2, Dak101,Irk5, Pho85, Pka1, Sch9, Hog1, Met3,

Pdk1/Pkh202*, Yck2*, Arg5,6*, Mpk1*, Mps1*, Kic1*, Bud32*, Bck1*, Utr1*, Fbp26*, Pos5*, Mec1*, Ipk1*, Swe102*, $\mathrm{Cdc7}^{\star}, \mathrm{Gsk3}^{\star}$, Ire1*, Vps15*, Ypk1*, Pdr802, Hob1, Sre1, Gat201, Fzc1, Fzc9, Fzc31, Nrg1*, Bzp1(Hxl1)*
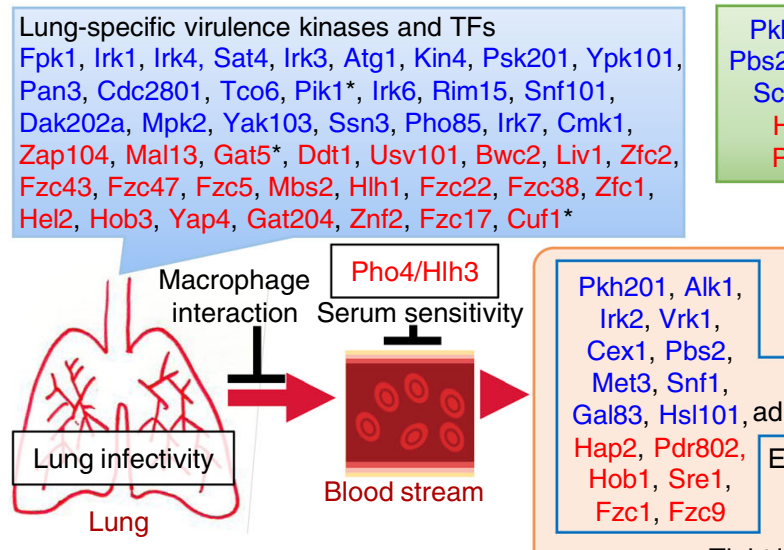

Tight junction

Pkh201, Alk1, Irk2, Vrk1, Cex1,

Pbs2, Yfh701, Met3, Hsl101, Snf1,

Sch9, Irk5, Gal83, Urk1, Ada2,

Hap2, Pdr802, Hob1, Sre1,

Pho4/HIh3, Jjj1, Fzc1, Fzc9

BBB Pkh201, Alk1, Irk2, Vrk1,
: Kinase, TF

*growth defect in

body temperature
Color code

TIk1, Trm7, Crk1, Mak3201,

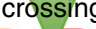

$\rightarrow$ Gal83, Kin1, Urk1, Hsl101,

Yak1, Snf1, Ssk2, Hog1,

Met3, Yck2*, Arg5,6*, Mpk1*,

Mps $1^{\star}$, Kic1*, Bud32*, Bck1*,

Utr1 $^{*}, \mathrm{Fbp}^{*} 6^{*}, \mathrm{Pos}^{\star}, \mathrm{Mec}^{*}$

Ipk1*, Swe102*, Ada2, Hap2,

Pdr802, Hob1, Sre1, Zfc3,

Gat201, Bzp2*, Nrg1*

Survival in the brain parenchyma

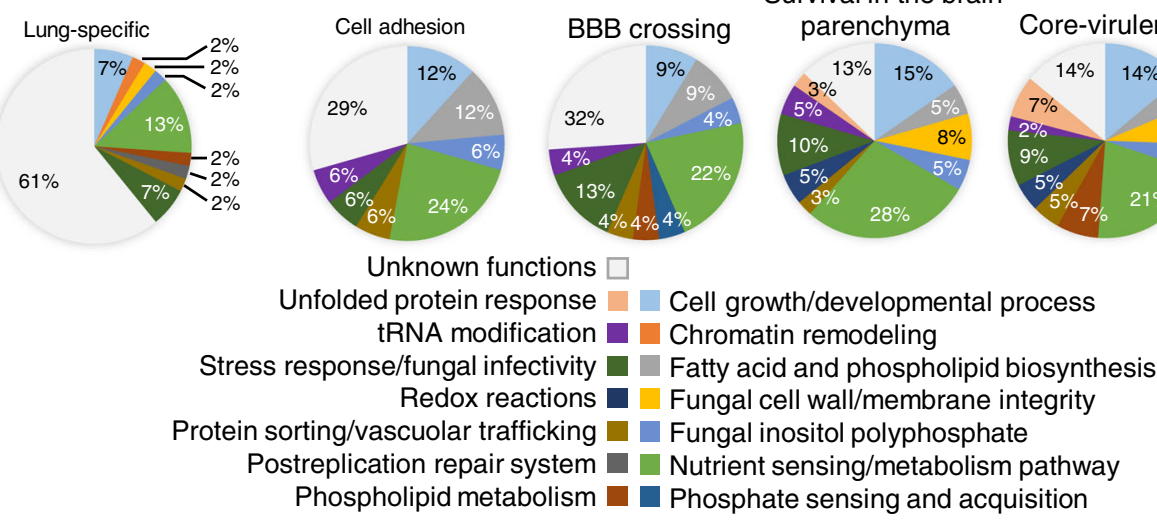

Fig. 7 The signalling network regulating brain infection in Cryptococcus neoformans. Gene Ontology (GO) term-based categorisation of lung-infectionrelated, brain-infection-related, and core-virulence kinases and TFs, which are summarised in Supplementary Data 2 and 3, are illustrated. Asterisk ( ${ }^{*}$ ) indicates gene deletion mutants that exhibit growth defects at mammalian body temperature. Blue and red letters denote kinases and TFs, respectively, involved in the virulence of $C$. neoformans. Bold letters indicate kinases and TFs that play important roles in both BBB crossing and survival in brain parenchyma.

may have a role downstream of Ypk1 ${ }^{15}$. Ypk1 is known to modulate the plasma membrane integrity in the TORC2 signalling pathway in S. cerevisiae ${ }^{37}$. In C. neoformans, we found that Ypk1 was required for both lung and brain infections. Therefore, Fpk1 could be responsible for a subset of Ypk1-dependent functions, which are likely to promote the lung infection of $C$. neoformans. Due to its potential role in maintaining the plasma membrane integrity, Fpk1 may be involved in the interaction of C. neoformans with lung alveolar macrophages. Our preliminary data demonstrate that Fpk1 indeed promotes inhibition of phagosome maturation within macrophages phagocytising C. neoformans (unpublished data).

Here, we found that $C$. neoformans utilises complex signalling pathways involved in diverse biological processes to infect the brain as summarised in Fig. 7. For BBB crossing, the following biological processes are involved: cell cycle regulation (Alk1 and Hsl101), tRNA export (Cex1), endoplasmic membrane assembly (Yfh701), stress response and adaptation (Pbs2), lipid and sterol metabolism and related regulations (Pkh201, Sre1, and Hob1), haeme-mediated respiration control (Hap2), ribosome biogenesis (Vrk1, Sch9 and Jjj1), carbon source utilisation (Gal83 and Snf1), methionine metabolism (Met3), histone acetylation and capsule biosynthesis (Ada2), phosphate sensing and metabolism (Pho4), Tor signalling (Sch9), and unknown functions (Irk2, Irk5, Fzc1, Fzc9, and Pdr802). For survival in the brain parenchyma, the following biological processes are involved: glucose sensing and metabolism (Snf1, Gal83, Yck2, Fpb26, Yak1, and Nrg1), amino acid metabolism (Arg5,6 and Met3), polarised exocytosis (Kin1), pyrimidine ribonucleotide salvage pathway (Urk1), mitochondrial redox reactions (Pos5 and Utr1), tRNA modification (Trm7 and Bud32), replication or maintenance of double stranded RNAcontaining particles (Mak3201), meiosis activation (Crk1, yeast Ime2 ortholog), cell cycle and morphology regulation (Mec1, Mps1, Alk1, Swe102, and Hsl101), stress response and adaptation (Ssk2 and Hog1), lipid-mediated regulations (Pkh201), histone acetyltransferase activity (Ada2), haeme-mediated respiration control (Hap2), ribosome biogenesis (Vrk1), Inositol polyphosphate biosynthetic process (Ipk1), sterol biosynthesis pathway (Sre1 and Hob1), cell wall/membrane integrity (Kic1, Bck1 and Mpk1), virulence factor regulation (Gat201) and unknown functions (Bzp2, Tlk1, Zfc3, Irk2, and Pdr802). Among these, 4 kinases (Pkh201, Alk1, Irk2 and Vrk1) and 5 TFs (Ada2, Hap2, Sre1, Hob1, and Pdr802) were required for both BBB crossing and survival in the brain parenchyma, indicating that lipid-mediated 
regulation, cell cycle regulation, chromatin remodelling, and haeme-mediated respiration control are critical for both BBB crossing and survival inside the brain. Collectively, these findings indicate that $C$. neoformans employs complex signalling networks governing diverse biological processes to promote $\mathrm{BBB}$ crossing and survival/proliferation in the brain parenchyma.

Previously known brain infection factors include two inositol transporters (Itr1a and Itr3c) ${ }^{38}$, hyaluronic acid synthase $(\mathrm{Cps} 1)^{39}$, metalloprotease $(\mathrm{Mpr} 1)^{31}$, urease $(\mathrm{Ure} 1)^{40,41}$, phospholipase $(\mathrm{Plb} 1)^{42}, \mathrm{Fnx}^{43}$, and Rub1 ${ }^{43}$. Liu et al. demonstrated that addition of external inositol induces expression of CPS1 in C. neoformans and enhances production of hyaluronic acid that facilitates the binding of the pathogen to CD44 glycoprotein in $\mathrm{HBMECs}^{38}$. In support, our in vivo transcriptional profiling analysis showed that expression of ITR1A, ITR3C, MPR1, CPS1, $P L B 1$, and FNX1 was indeed upregulated during brain infection, although their expression was often induced in other infected tissues (lungs, kidneys, and spleens). In addition to these known brain-infection factors, we found that redundant and distinct sets of kinases and TFs are involved in each step of brain infection of C. neoformans: adhesion to HMBECs, traversing the BBB, and proliferation in the brain parenchyma. Biological functions of genes involved in $\mathrm{BBB}$ adhesion and crossing are highly correlated and include nutrient sensing/metabolism, fatty acid/phospholipid biosynthesis, vacuolar trafficking, and inositol signalling. Notably, fatty acid/phospholipid biosynthesis and vacuolar trafficking are more important for BBB adhesion and crossing than for survival in the brain parenchyma. In contrast, genes involved in nutrient sensing and metabolism appeared to be most important for survival in the brain parenchyma, which supports the previous finding that the non-fermentative gluconeogenic pathway and the fermentative glycolytic pathway are required for the initial lung infection and the subsequent brain infection, respectively, of $C$. neoformans ${ }^{44}$. Besides those required for the growth at $37^{\circ} \mathrm{C}$, lung infection or BBB adhesion/crossing, several kinases and TFs (Tlk1, Trm7, Crk1, and Mak3201) appeared to be specifically required for the survival in the brain parenchyma (Fig. 7). Notably, Tlk1 encodes a Tor-like kinase, but its biological functions remain unclear. Although orthologous to Tor1, it does not bind to rapamycin and its deletion does not result in any evident in vitro phenotypic changes ${ }^{15,45}$. As Tor 1 is involved in nutrient sensing pathway, it is conceivable that Tlk1 may play a role in sensing some brain-specific nutritional condition for $C$. neoformans.

Among the brain-infection-related kinases/TFs, four kinases (Pkh201, Alk1, Irk2, and Vrk1) and 4 TFs (Hap2, Pdr802, Sre1, and Hob1) were found to be required for all three brain infection steps (BBB adhesion/crossing and survival in the brain parenchyma), strongly indicating that they are key regulators of $C$. neoformans brain infection. Notably, here we found that Hobl controlled the HMC-induced expression of ITR1A, ITR3C, and $M P R 1$ as well as other brain-infection-related TFs, including FZC31, PDR802, SRE1, and PHO4, further confirming that the homeobox TF is a critical transcriptional regulator of $C$. neoformans brain infection. The master regulatory role of Hob1 in brain infection of $C$. neoformans was further manifested by its dispensable role in C. deuterogattii (R265), which mainly targets the lungs instead of brain ${ }^{33}$. Our data showed that both H99 and R265 strains are able to cross the BBB, although the former strain does more efficiently than the latter strain. Notably, deletion of $H O B 1$ significantly impaired the ability of the H99 strain, but not the R265 strain, to cross the BBB. Our allele-swapping experiments demonstrated that the difference in Hob1 sequence was not responsible for the distinct roles of Hob1 in the two pathogenic Cryptococcus strains. There could be several explanations for this finding. Hob1 downstream target proteins may function differently in the two species. Another possibility is that a Hob1binding element could be differentially distributed in the promoter regions of Hob1 downstream targets. All these possibilities could be addressed in future RNA-seq and chromatin IP-seq analyses.

The current analyses have the following limitations. First, we could not track down the genes involved in neurotropism of $C$. neoformans, due to a lack of proper known systems. It has been hypothesised that $C$. neoformans has a neurotropism to the CNS to utilise neurotransmitters ${ }^{13}$. Therefore, some of the kinase and TF mutants may have reduced brain-STM scores because of a lack of neurotropism. Second, the in vitro HBMECs-based BBB transwell system used in this study is distinct from the natural, dynamic BBB structure, which consists of HBMECs, pericytes, and brain astrocytes ${ }^{12}$. Therefore, some of the kinase and TF mutants could be defective in crossing the natural $\mathrm{BBB}$. The role of these signalling components in brain infection should be further dissected in future studies. Regardless of these limitations, this study allows us to understand complex signalling networks governing the whole infection processes of C. neoformans and provide therapeutic targets for treating fungal meningoencephalitis.

\section{Methods}

Ethics statement. Animal care and all experiments were conducted in accordance with the ethical guidelines of the Institutional Animal Care and Use Committee (IACUC) of Yonsei University and National Taiwan University. The Yonsei University and National Taiwan University IACUC approved all of the vertebrate studies.

The STM-based murine infectivity assay. The high-throughput murine braininfectivity test was performed using the previously published TF and kinase mutant libraries with the nourseothricin acetyltransferase (NAT) selection marker containing 46 unique signature tags (four and five groups of the TF and kinase mutant libraries, respectively) ${ }^{15,16}$ (Supplementary Data 4 ). The ste50 $\Delta$ mutants were used as virulent control strains, as previously reported ${ }^{16,23}$. In a given group of mutants with unique signature tags, each mutant was grown at $30^{\circ} \mathrm{C}$ in YPD medium for $16 \mathrm{~h}$ separately and washed three times with phosphate-buffered saline (PBS). The concentration of each mutant was adjusted to $10^{7}$ cells per $\mathrm{ml}$ and $50 \mu \mathrm{l}$ of each mutant sample was pooled into one tube. For preparation of the input genomic DNA, $200 \mu \mathrm{l}$ of the mutant pool was spread on YPD plate, incubated at $30^{\circ} \mathrm{C}$ for 3 days, and scraped. For preparation of the output genomic DNA samples, $50 \mu \mathrm{l}$ of the mutant pool $\left(5 \times 10^{5}\right.$ cells per mouse) was infected into seven-week-old female $\mathrm{A} / \mathrm{J}$ mice (Japan SLC, Inc.) through intravenous or intracerebroventricular (ICV) injection. For intravenous injection, the tail was placed in warm $\left(40^{\circ} \mathrm{C}\right)$ water for expanding the vein and the tail vein injection was performed in restrained mice ( 3 mice per pooled mutant group). For ICV injection, mice were anaesthetised with $2 \%$ tribromoethanol by intraperitoneal injection $\left(20 \mathrm{ml} \mathrm{kg}^{-1}\right.$, Sigma Aldrich) and placed on a stereotaxic device (David Kopf Instruments). The control strain and mutant pool were unilaterally injected into the ventricle (anteroposterior,

$-0.2 \mathrm{~mm}$; lateral, $-1.0 \mathrm{~mm}$; ventral $-2.0 \mathrm{~mm}$ ) using a NanoFil needle (WPI) with Hamilton syringe and pump (WPI). The infected mice were sacrificed after $7 \mathrm{dpi}$ and their brains were harvested. The recovered brains were homogenised in $5 \mathrm{ml}$ PBS, $200 \mu \mathrm{l}$ of them was spread onto the YPD plates containing $100 \mu \mathrm{g}$ per $\mathrm{ml}$ of chloramphenicol, incubated at $30^{\circ} \mathrm{C}$ for 2 days, and scraped. Total genomic DNA was extracted from scraped input and output samples by the cetrimonium bromide (CTAB) method. Quantitative PCR was performed with the tag-specific primers, as previously reported ${ }^{15}$, using CFX96 ${ }^{\mathrm{TM}}$ Real-Time PCR system (Bio-Rad). The STM score was calculated as previously described ${ }^{16}$. Briefly, relative changes in genomic DNA amounts were calculated by the $2^{-\Delta \Delta C T}$ method to determine the STM score. If the STM locus-tag was not detected in the output samples by qPCR, the amount of DNA was considered to be very small, and the maximum cycle of qPCR, 40, was used as the $\mathrm{Ct}$ value. The mean fold-changes in input versus output samples were

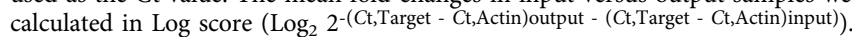
STM scores were measured for two independent mutant strains for each kinase and TF mutant (Supplementary Fig. 2).

NanoString in vivo transcription profiling analysis. Six-week-old female A/J mice were infected with $5 \times 10^{5}$ cells through nasal inhalation. Each group of 3 mice was sacrificed after $3,7,14$ or 21 dpi. The lungs, brain, spleens and kidneys were recovered and lyophilised. Dried organs were homogenised and total RNA was extracted by using RNA extraction kit (easy-BLUE, Intron Biotechnology). Samples containing $10 \mathrm{ng}$ of total RNA isolated from C. neoformans grown under in vitro basal conditions $\left(30^{\circ} \mathrm{C}\right.$; YPD medium) or $10 \mu \mathrm{g}$ of total RNA isolated from 
C. neoformans-infected mouse tissues were reacted with the designed NanoString probe code set and incubated at $65^{\circ} \mathrm{C}$ overnight $(12$ to $18 \mathrm{~h}$ ). The hybridised samples were processed on a NanoString prep station according to the manufacturer's standard protocol of the nCounter ${ }^{\oplus}$ platform, as previously reported ${ }^{26,46}$. Scanning was performed by digital analyser through high resolution (600 fields) option and normalised by nSolver software (provided by NanoString). A total of eight housekeeping genes were used for expression normalisation, including those used in a previous paper ${ }^{47}$ (mitochondrial protein, CNAG_00279; microtubule binding protein, CNAG_00816; aldose reductase, CNAG_02722; cofilin, CNAG_02991; actin, CNAG 00483; tubulin $\beta$ chain, CNAG_01840; tubulin $\alpha-1 \mathrm{~A}$ chain, CNAG_03787; histone H3, CNAG_04828). The normalised data was transformed to $\log _{2}$ score to express the fold change and subject to clustering using one minus Pearson correlation with average linkage by Morpheus (https://software. broadinstitute.org/morpheus).

\section{Construction of gene deletion and complementation mutants. The mprls} mutant was reconstructed by double-joint PCR using $N A T^{\mathrm{r}}$-split marker and biolistic transformation (Supplementary Fig. 5), as previously described ${ }^{15}$. Briefly, $600 \mu \mathrm{g}$ of gold microcarrier beads $(0.6 \mu \mathrm{m}$, Bio-Rad) were coated with the PCRamplified gene disruption cassettes and introduced into the $C$. neoformans cells by using particle delivery system (PDS-100, Bio-Rad). The transformed cells were further incubated at $30^{\circ} \mathrm{C}$ for recovery of cell membrane integrity, scrapped after $4 \mathrm{~h}$, and further incubated on YPD containing nourserothricin for selection. Hob1 orthologues in C. deuterogattii R265 (CNBG_5938) and C. deneoformans XL280 strains (CNC05440) and Sre1 orthologues in R265 (CNBG_9608) and XL280 (CNJ02310) were disrupted by double-joint PCR using NAT ${ }^{\text {- }}$ split marker and the primer sets presented in Supplementary Data 5 and biolistic transformation. Stable transformants were selected on YPD medium containing nourserothricin and screened by diagnostic PCR. The correct genotypes of screened mutants were confirmed by Southern blot analyses (Supplementary Fig. 6). To verify the

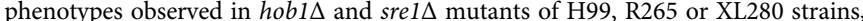
we constructed corresponding complementary strains (Supplementary Fig. 7) as previously described ${ }^{48}$. Briefly, PCR fragments containing the $5^{\prime}$ - and $3^{\prime}$-flanking regions and the open reading frames of $H O B 1$ and SRE1 genes in each background strain were amplified with gene-specific primers (Supplementary Data 5). The amplified PCR products were cloned into the plasmid pTOP-V2 to confirm the DNA sequence. Due to the long length of each SRE1 gene, we cloned the 5'fragments of each SRE1 gene using primer 1 and primer 2 (XmaI-SacII ends for H99, XbaI-Nhe1 ends for XL280, and XmaI-XhoI ends for R265) and the 3'fragments using primer 3 and primer 4 (SacII-NotI ends for H99, Nhe1-ApaI ends for XL280, and XhoI-NotI ends for R265) listed in Supplementary Data 5. Each 3'fragment was subcloned into the $5^{\prime}$-fragment in pTOP-V2, and their sequences were confirmed. The cloned whole genes were subcloned into the plasmid pJAF12 or pNEO vector containing neomycin-resistance gene (NEO) selection marker to produce plasmid pJAF12-HOB1 or pNEO-SRE1 in each species. The designated restriction enzyme-digested plasmid (AfeI for pJAF12-H99-HOB1, SwaI for pJAF12-R265-HOB1, MfeI for pJAF12-XL280-HOB1, AgeI for pNEO-H99-SRE1, Nsil for pJAF12-R265-SRE1, and HpaI for pNEO-XL280-SRE1) were linearised and biolistically delivered into the hobl $\Delta$ and srel $\Delta$ mutants in each species. The re-integration of HOB1 or SRE1 into their native loci was confirmed by diagnostic PCR with specific primer sets presented in Supplementary Data 5. To construct the HOB1 allele-swapping strain, the $C$. deuterogatti R265 HOB1 ORF was inserted between C. neoformans $\mathrm{H} 99$ HOB1 native promoter and terminator as follows (See schematic diagram in Supplementary Fig. 9b). The $5^{\prime}$-fragment (native promoter region of $\mathrm{H} 99 \mathrm{HOB1}$ ) was amplified with specific primers, cloned into pTOP-V2 vector and sequenced. The $3^{\prime}$-fragment (R265 HOB1 open reading frame with the native terminator region of $\mathrm{H} 99 \mathrm{HOB1}$; NdeI-NsiI ends) was synthesized (Cosmo Genetech, Korea), cloned into pTOP-V2 vector, and then subcloned into the 5'fragment (NotI-NdeI ends) in pTOP-V2. The whole insert was subcloned into the pNEO vector containing $N E O$ selection marker to produce plasmid pNEO$\mathrm{HOB}_{1}{ }^{\mathrm{R} 265}$. The plasmid was linearised by Afe1 and biolistically introduced into the

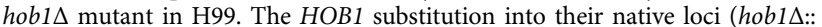
$\mathrm{HOB1}^{\mathrm{R} 265}$ ) was confirmed by diagnostic PCR with specific primer sets (Supplementary Data 5).

In vitro BBB crossing and adhesion assays. Human brain microvascular endothelial cell (HBMEC) line (hCMEC/D3 cell line, Merck) was cultured performed as previously described ${ }^{30,32}$. Briefly, the $5 \times 10^{4} \mathrm{hCMEC} / \mathrm{D} 3$ cells in EGM ${ }^{\mathrm{st}}-2$ medium (Lonza) were seeded on collagen (Corning) coated $8 \mu \mathrm{m}$-porous membranes (BD Falcon) for BBB crossing assay or 12-well plates (BD Falcon) for BBB adhesion assay. The day after seeding, medium was replaced with a fresh EGM ${ }^{\mathrm{m}}-2$ medium supplemented with $2.5 \%$ human serum, and further grown for 4 days. A day before yeast inoculation, the medium was replaced with $0.5 \times$ diluted $\mathrm{EGM}^{\mathrm{m}}-2$ medium and the cells were maintained at $37^{\circ} \mathrm{C}$ and $5 \% \mathrm{CO}_{2}$ incubator. Integrity of tight junctions between hCMEC/D3 cells was confirmed by measuring the transendothelial electrical resistance (TEER), which should be around $200 \Omega$ per $\mathrm{cm}^{2}$. TEER was measured by Epithelial Volt per Ohm Metre (EVOM ${ }^{2}$ device, World Precision Instruments). For BBB crossing assay, $5 \times 10^{5}$ cells of $C$. neoformans WT (H99), TF/kinase deletion mutants, or S. cerevisiae WT (S288C strain) were added to $500 \mu \mathrm{l}$ of PBS and inoculated onto the top of the porous membranes. After $24 \mathrm{~h}$ incubation at $37^{\circ} \mathrm{C}$ in $5 \% \mathrm{CO}_{2}$ incubator, the number of yeast cells passing through the porous membrane was measured by counting CFU. TEER was measured by using $\mathrm{EVOM}^{2}$ device before and after inoculation of yeast cells. The BBB migration ratio was calculated by dividing the output $\mathrm{CFU}$ of each tested strain by that of WT. For BBB adhesion assay, $5 \times 10^{5}$ yeast cells in $100 \mu \mathrm{l}$ of PBS were inoculated onto a monolayer of hCMEC/D3 cells grown in a 12-well plate, and incubated for $24 \mathrm{~h}$ at $37^{\circ} \mathrm{C}$ in $5 \% \mathrm{CO}_{2}$ incubator. Following incubation, cultures were washed 3 times with PBS and incubated with sterile distilled water for $30 \mathrm{~min}$ at $37^{\circ} \mathrm{C}$ incubator to burst the hCMEC/D3 cells, and collected for CFU counting. The BBB adhesion ratio was calculated by dividing the adhered CFU of each test strain by that of WT C. neoformans.

Expression analysis. C. neoformans $\mathrm{H} 99$ strain and deletion mutants were incubated in YPD broth at $30^{\circ} \mathrm{C}$ for $16 \mathrm{~h}$, and each strain was sub-inoculated into $50 \mathrm{ml}$ of fresh YPD broth and further incubated until the optical density of the medium at $600 \mathrm{~nm}\left(\mathrm{OD}_{600}\right)$ reached the value of 0.8 . The culture was separated into two-tubes ( $25 \mathrm{ml}$ each), centrifuged, and washed three times with sterile distilled water. One tube was kept in liquid nitrogen tank to monitor basal expression levels, and the other tube was resuspended with equal volume of RPMI1640 medium containing $10 \% \mathrm{FBS}$. After $3 \mathrm{~h}$ incubation at $37^{\circ} \mathrm{C}$ in a $\mathrm{CO}_{2}$ incubator with $120 \mathrm{rpm}$ horizontal shaking, cells were centrifuged and all samples were lyophilised overnight. Total RNA was extracted by using RNA extraction kit (easy-BLUE, Intron Biotechnology) and cDNA was synthesized using a Reverse Transcriptase kit (Thermo Scientific). Real-time quantitative PCR was performed for the target gene and $A C T 1$; specific primer pairs are listed in Supplementary Data 5. The expression levels of the target gene were calculated by the threshold $2^{-\Delta \Delta C T}$ method.

Growth and chemical susceptibility assays. Cells grown overnight at $30^{\circ} \mathrm{C}$ were serially diluted tenfold ( 1 to $10^{4}$ dilution) and spotted on YPD plate containing the indicated concentrations of chemical agents as follows: Osmotic (sorbitol) and cation/salt stress $(\mathrm{NaCl}$ and $\mathrm{KCl})$ under either glucose-rich (YPD) or glucosestarved (YPD without dextrose; YP) condition; diamide (a thiol-specific oxidant) hydrogen peroxide $\left(\mathrm{H}_{2} \mathrm{O}_{2}\right)$, menadione (as superoxide anion generator), and tert butyl hydroproxide (an organic peroxide) for oxidative stress; methyl methanesulfonate (MMS) and hydroxyurea (HU) for genotoxic stress; tunicamycin (TM) and dithiothreitol (DTT) for ER stress; calcoflour white and Congo red for cell wall destabilising stress; sodium dodecyl sulfate (SDS) for membrane destabilising stress; amphothericin B, fluconazole, flucytosin, and fludioxonil for antifungal drug susceptibility. Cells were incubated at $30^{\circ} \mathrm{C}$ and photographed during a period of 2-4 days. To test the growth rate of each mutant at distinct temperature, the serially tenfold diluted (1 to $10^{4}$ dilution) cells spotted onto the YPD plates were incubated at $25^{\circ} \mathrm{C}, 30^{\circ} \mathrm{C}, 37^{\circ} \mathrm{C}$, and $39^{\circ} \mathrm{C}$, and photographed as above.

In vitro virulence-factor production assay. To perform capsule production assay, cells were cultured in YPD broth at $30^{\circ} \mathrm{C}$ overnight, and then spotted onto DME medium and incubated at $37^{\circ} \mathrm{C}$ for 2 days. After incubation, the capsules were stained with India ink and visualised with an Olympus BX51 microscope equipped with a SPOT insight digital camera. For quantitative analysis of capsule production, $10 \%$ formalin-fixed cells were adjusted to $3 \times 10^{8}$ cells per $\mathrm{ml}$ in phosphatebuffered saline (PBS), and injected $(50 \mu \mathrm{l})$ into microhaematocrit capillary tubes (Kimble Chase) in triplicates. All tubes were placed in an upright vertical position for 3 days to precipitate cells by gravity. The relative packed cell volume was measured with haematocrit capillary tubes by calculating the ratio of the lengths of the packed cell phase to the total phase (cells plus liquid phases), as previously described ${ }^{15}$. To examine melanin production, cells were grown overnight in YPD broth at $30^{\circ} \mathrm{C}$, then $5 \mu \mathrm{l}$ of each culture was spotted on Niger seed media containing $0.1 \%$ or $0.2 \%$ glucose, incubated at $30^{\circ} \mathrm{C}$ and photographed after $3-4$ days

Murine virulence assay of hob1s and sre1s mutants. Seven- to eight-week-old female C57BL/6 mice (BioLASCO Taiwan Co., Ltd) or A/J mice (Japan SLC, Inc.) were used to produce the murine intranasal infection model. C. neoformans or $C$ deuterogattii strains were cultured in YPD broth overnight at $30{ }^{\circ} \mathrm{C}$ with shaking at $200 \mathrm{rpm}$, and washed twice with PBS. Cells were counted with hemocytometer, resuspended in PBS, and diluted to $10^{6}$ cells (for C57BL/6 mice) or $10^{7}$ cells (for A/ $\mathrm{J}$ mice) per ml. Appropriate dilutions of the cells were plated onto YPD agar plate and incubated at $30^{\circ} \mathrm{C}$ for $48 \mathrm{~h}$ in order to confirm CFU numbers and viability. Groups of $10(\mathrm{C} 57 \mathrm{BL} / 6)$ or $7(\mathrm{~A} / \mathrm{J})$ mice were anesthetised with isoflurane (Panion $\&$ BF Biotech Inc.) for C57BL/6 mice or $2 \%$ tribromoethanol (T48402, SigmaAldrich) for A/J mice, and inoculated with $C$. neoformans or C. deuterogattii strains via intranasal instillation of inocula of $5 \times 10^{4}$ cells (for C57BL/ 6 mice) or $5 \times 10^{5}$ cells (for A/J mice) in $50 \mu \mathrm{l}$ PBS. The survival of mice was monitored twice daily for 49 days, and moribund mice (mice that were unable to eat or drink, their body weight was reduced by $15 \%$, or were hunched) were euthanised with $\mathrm{CO}_{2}$. The survival curve was created with Prism 8 . The significance of differences was determined using Log-rank (Mantel-Cox) test.

Statistical analysis. Statistical analyses were performed with GraphPad Prism version 8. For the murine STM analysis, we used one-way analysis of variance (ANOVA) employing Bonferroni correlation to STM scores of ste50D (control) and 
knockout mutants (cutoff $>2$ or $<-2$ ) with three mice per STM group. In addition, the STM score of the second independent mutants was measured and calculated in the same manner in three independent mice. For the BBB crossing, adhesion, and gene expression assay, we used a two-tailed unpaired Student's $t$-test to compare the two groups. For the multiple comparison between the two-species of C. neoformans and C. deuterogattii, we used one-way ANOVA with Bonferroni's post correction. The murine survival assay was determined by using Log-rank (MantelCox) test. For all analysis, $P<0.05$ was considered significant and the error bars indicated \pm standard error of the mean (SEM) from three or more independent experiments.

Reporting summary. Further information on research design is available in the Nature Research Reporting Summary linked to this article.

\section{Data availability}

STM-based murine data for all kinase and TF mutants are provided in Supplementary Figs. 1 and 2. For the NanoString-nCounter ${ }^{\circledR}$ analysis, in vivo kinase and TF gene expression data and probe information are included in Supplementary Data 1. All the sequence and protein domain information was obtained from FungiDB (https://fungiDB.org) and UniProtKB (https://www.uniprot.org/), respectively. The source data underlying Supplementary Figs 5, 6, and 7 are provided as a Source Data file. Other data supporting the findings of this study are available from the corresponding authors upon request.

Received: 19 June 2019; Accepted: 3 March 2020;

Published online: 23 March 2020

\section{References}

1. Fisher, M. C. et al. Emerging fungal threats to animal, plant and ecosystem health. Nature 484, 186-194 (2012).

2. Brown, G. D. et al. Hidden killers: human fungal infections. Sci. Transl. Med. 4, 165rv113 (2012)

3. Rajasingham, R. et al. Global burden of disease of HIV-associated cryptococcal meningitis: an updated analysis. Lancet Infect. Dis. 17, 873-881 (2017).

4. Kwon-Chung, K. J. et al. The case for adopting the "Species Complex" nomenclature for the etiologic agents of cryptococcosis. mSphere 2, e00357-16 (2017).

5. Zaragoza, O. et al. The capsule of the fungal pathogen Cryptococcus neoformans. Adv. Appl. Microbiol. 68, 133-216 (2009).

6. Bojarczuk, A. et al. Cryptococcus neoformans intracellular proliferation and capsule size determines early macrophage control of infection. Sci. Rep. 6, 21489 (2016)

7. Wang, Y., Aisen, P. \& Casadevall, A. Cryptococcus neoformans melanin and virulence: mechanism of action. Infect. Immun. 63, 3131-3136 (1995).

8. Mednick, A. J., Nosanchuk, J. D. \& Casadevall, A. Melanization of Cryptococcus neoformans affects lung inflammatory responses during cryptococcal infection. Infect. Immun. 73, 2012-2019 (2005).

9. Ma, H., Croudace, J. E., Lammas, D. A. \& May, R. C. Expulsion of live pathogenic yeast by macrophages. Curr. Biol. 16, 2156-2160 (2006)

10. Johnston, S. A. \& May, R. C. Cryptococcus interactions with macrophages: evasion and manipulation of the phagosome by a fungal pathogen. Cell Microbiol. 15, 403-411 (2013).

11. Johnston, S. A. \& May, R. C. The human fungal pathogen Cryptococcus neoformans escapes macrophages by a phagosome emptying mechanism that is inhibited by Arp2/3 complex-mediated actin polymerisation. PLoS Pathog. 6, e1001041 (2010)

12. Dziegielewska, K. M., Ek, J., Habgood, M. D. \& Saunders, N. R. Development of the choroid plexus. Microsc. Res. Tech. 52, 5-20 (2001).

13. Esher, S. K., Zaragoza, O. \& Alspaugh, J. A. Cryptococcal pathogenic mechanisms: a dangerous trip from the environment to the brain. Mem. Inst. Oswaldo Cruz 113, e180057 (2018).

14. Griffiths, E. J., Kretschmer, M. \& Kronstad, J. W. Aimless mutants of Cryptococcus neoformans: failure to disseminate. Fungal Biol. Rev. 26, 61-72 (2012).

15. Lee, K. T. et al. Systematic functional analysis of kinases in the fungal pathogen Cryptococcus neoformans. Nat. Commun. 7, 12766 (2016)

16. Jung, K. W. et al. Systematic functional profiling of transcription factor networks in Cryptococcus neoformans. Nat. Commun. 6, 6757 (2015).

17. Bahn, Y. S., Hicks, J. K., Giles, S. S., Cox, G. M. \& Heitman, J. Adenylyl cyclase-associated protein Acal regulates virulence and differentiation of Cryptococcus neoformans via the cyclic AMP-protein kinase A cascade. Eukaryot. Cell 3, 1476-1491 (2004).

18. Alspaugh, J. A. et al. Adenylyl cyclase functions downstream of the Galpha protein Gpal and controls mating and pathogenicity of Cryptococcus neoformans. Eukaryot. Cell 1, 75-84 (2002).
19. Bahn, Y. S., Geunes-Boyer, S. \& Heitman, J. Ssk2 mitogen-activated protein kinase kinase kinase governs divergent patterns of the stress-activated Hog1 signaling pathway in Cryptococcus neoformans. Eukaryot. Cell 6, 2278-2289 (2007)

20. Bahn, Y. S., Kojima, K., Cox, G. M. \& Heitman, J. Specialization of the HOG pathway and its impact on differentiation and virulence of Cryptococcus neoformans. Mol. Biol. Cell 16, 2285-2300 (2005).

21. Gerik, K. J. et al. Cell wall integrity is dependent on the $P K C 1$ signal transduction pathway in Cryptococcus neoformans. Mol. Microbiol. 58, 393-408 (2005).

22. Kraus, P. R., Fox, D. S., Cox, G. M. \& Heitman, J. The Cryptococcus neoformans MAP kinase Mpk1 regulates cell integrity in response to antifungal drugs and loss of calcineurin function. Mol. Microbiol. 48, 1377-1387 (2003).

23. Cheon, S. A. et al. Unique evolution of the UPR pathway with a novel bZIP transcription factor, Hxll, for controlling pathogenicity of Cryptococcus neoformans. PLoS Pathog. 7, e1002177 (2011).

24. Liu, O. W. et al. Systematic genetic analysis of virulence in the human fungal pathogen Cryptococcus neoformans. Cell 135, 174-188 (2008).

25. Cramer, K. L., Gerrald, Q. D., Nichols, C. B., Price, M. S. \& Alspaugh, J. A. Transcription factor Nrg1 mediates capsule formation, stress response, and pathogenesis in Cryptococcus neoformans. Eukaryot. Cell 5, 1147-1156 (2006)

26. Xu, W., Solis, N. V., Filler, S. G. \& Mitchell, A. P. Pathogen gene expression profiling during infection using a NanoString nCounter platform. Methods Mol. Biol. 1361, 57-65 (2016).

27. Cadieux, B. et al. The mannoprotein Cig1 supports iron acquisition from heme and virulence in the pathogenic fungus Cryptococcus neoformans. J. Infect. Dis. 207, 1339-1347 (2013).

28. Jung, W. H., Hu, G., Kuo, W. \& Kronstad, J. W. Role of ferroxidases in iron uptake and virulence of Cryptococcus neoformans. Eukaryot. Cell 8, 1511-1520 (2009).

29. Lev, S. et al. Pho4 is essential for dissemination of Cryptococcus neoformans to the host brain by promoting phosphate uptake and growth at alkaline $\mathrm{pH}$. mSphere 2, e00381-16 (2017).

30. Vu, K., Weksler, B., Romero, I., Couraud, P. O. \& Gelli, A. Immortalized human brain endothelial cell line hCMEC/D3 as a model of the blood-brain barrier facilitates in vitro studies of central nervous system infection by Cryptococcus neoformans. Eukaryot. Cell 8, 1803-1807 (2009).

31. $\mathrm{Vu}, \mathrm{K}$. et al. Invasion of the central nervous system by Cryptococcus neoformans requires a secreted fungal metalloprotease. mBio 5, e01101-01114 (2014).

32. Santiago-Tirado, F. H., Onken, M. D., Cooper, J. A., Klein, R. S. \& Doering, T. L. Trojan horse transit contributes to blood-brain barrier crossing of a eukaryotic pathogen. mBio 8, e02183-02116 (2017).

33. Ngamskulrungroj, P., Chang, Y., Sionov, E. \& Kwon-Chung, K. J. The primary target organ of Cryptococcus gattii is different from that of Cryptococcus neoformans in a murine model. mBio 3, e00103-00112 (2012).

34. Bielska, E. \& May, R. C. What makes Cryptococcus gattii a pathogen? FEMS Yeast Res. 16, fov106 (2015).

35. Fraser, J. A. et al. Same-sex mating and the origin of the Vancouver Island Cryptococcus gattii outbreak. Nature 437, 1360-1364 (2005).

36. Ngamskulrungroj, P., Price, J., Sorrell, T., Perfect, J. R. \& Meyer, W. Cryptococcus gattii virulence composite: candidate genes revealed by microarray analysis of high and less virulent Vancouver island outbreak strains. PLoS ONE 6, el6076 (2011).

37. Roelants, F. M., Leskoske, K. L., Martinez Marshall, M. N., Locke, M. N. \& Thorner, J. The TORC2-dependent signaling network in the yeast Saccharomyces cerevisiae. Biomolecules 7, pii: E66 (2017).

38. Liu, T. B. et al. Brain inositol is a novel stimulator for promoting Cryptococcus penetration of the blood-brain barrier. PLoS Pathog. 9, e1003247 (2013).

39. Jong, A. et al. Identification and characterization of CPS1 as a hyaluronic acid synthase contributing to the pathogenesis of Cryptococcus neoformans infection. Eukaryot. Cell 6, 1486-1496 (2007).

40. Cox, G. M., Mukherjee, J., Cole, G. T., Casadevall, A. \& Perfect, J. R. Urease as a virulence factor in experimental cryptococcosis. Infect. Immun. 68, 443-448 (2000).

41. Olszewski, M. A. et al. Urease expression by Cryptococcus neoformans promotes microvascular sequestration, thereby enhancing central nervous system invasion. Am. J. Pathol. 164, 1761-1771 (2004).

42. Cox, G. M. et al. Extracellular phospholipase activity is a virulence factor for Cryptococcus neoformans. Mol. Microbiol. 39, 166-175 (2001).

43. Tseng, H. K. et al. Identification of genes from the fungal pathogen Cryptococcus neoformans related to transmigration into the central nervous system. PLoS ONE 7, e45083 (2012)

44. Price, M. S. et al. Cryptococcus neoformans requires a functional glycolytic pathway for disease but not persistence in the host. mBio 2, e00103-0011 (2011).

45. Lee, H., Khanal Lamichhane, A., Garraffo, H. M., Kwon-Chung, K. J. \& Chang, Y. C. Involvement of PDK1, PKC and TOR signalling pathways in 
basal fluconazole tolerance in Cryptococcus neoformans. Mol. Microbiol 84, 130-146 (2012).

46. Fanning, S. et al. Divergent targets of Candida albicans biofilm regulator Bcr1 in vitro and in vivo. Eukaryot. Cell 11, 896-904 (2012).

47. O'Meara, T. R. et al. The Cryptococcus neoformans Rim101 transcription factor directly regulates genes required for adaptation to the host. Mol. Cell Biol. 34, 673-684 (2014)

48. Jung, K. W. et al. Evolutionarily conserved and divergent roles of unfolded protein response (UPR) in the pathogenic Cryptococcus species complex. Sci. Rep. 8, 8132 (2018).

\section{Acknowledgements}

This work was supported by National Research Foundation (NRF) grants funded by the Korean government (MSIT) (2016R1E1A1A01943365 and 2018R1A5A1025077 to Y.-S. B.; 2017R1A2B3011098 and 2017M3C7A1023471 to E.C; 2018R1C1B6009031 to K.-T.L.) and by the Strategic Initiative for Microbiomes in Agriculture and Food funded by Ministry of Agriculture, Food and Rural Affairs (918012-4 to Y.-S.B.). This work was also supported by the Korea Health Technology R\&D Project through the Korea Health Industry Development Institute (KHIDI) funded by the Ministry of Health and Welfare (HI18C1664 to E.C.), the Brain Korea 21 (BK21) PLUS program, and by AmtixBio, Co., Ltd. (to Y.-S.B.).

\section{Author contributions}

Y.-S.B. conceived the project. K.-T.L., J.H., D.-G.L., M.L., S.C., Y.-G.L., K.-W.J., A.H., Y.L., and S.-J.Y. performed experiments and analysed the data. K.-T.L., J.H., D.-G.L., Y.-L.C., J.-S.L., E.C., and Y.-S.B. supervised the experimental analysis. K.-T.L., J.H., D.-G.L., M.L., S.C., Y.-G.L., K.-W.J., A.H., Y.L., S.-J.Y., Y.-L.C., J.-S.L., E.C., and Y.-S.B. wrote the manuscript. All authors reviewed and approved this manuscript.

\section{Competing interests}

Yonsei University and AmtixBio, Co., Ltd. have jointly filed a patent application (patent No. 10-2019-0114797), on which K.T.L., J.H., D.-G.L, J.-S.L, E.C., and Y.-S.B. are listed as inventors. Y.-S.B. and J.-S.L. are scientific co-founder and chief executive officer, respectively, of AmtixBio, Co., Ltd. E.C., Y.-S.B., and J.-S.L. are stock holders of AmtixBio, Co., Ltd. AmtixBio, one of the funders, played a role in the conceptualisation of this manuscript. All other authors declare no competing interests.

\section{Additional information} Supplementary information is available for this paper at https://doi.org/10.1038/s41467020-15329-2.

Correspondence and requests for materials should be addressed to E.C. or Y.-S.B.

Peer review information Nature Communications thanks Julianne Djordjevic, Floyd Wormley and the other, anonymous, reviewer for their contribution to the peer review of this work. Peer reviewer reports are available.

Reprints and permission information is available at http://www.nature.com/reprints

Publisher's note Springer Nature remains neutral with regard to jurisdictional claims in published maps and institutional affiliations.

(c) (i) Open Access This article is licensed under a Creative Commons Attribution 4.0 International License, which permits use, sharing, adaptation, distribution and reproduction in any medium or format, as long as you give appropriate credit to the original author(s) and the source, provide a link to the Creative Commons license, and indicate if changes were made. The images or other third party material in this article are included in the article's Creative Commons license, unless indicated otherwise in a credit line to the material. If material is not included in the article's Creative Commons license and your intended use is not permitted by statutory regulation or exceeds the permitted use, you will need to obtain permission directly from the copyright holder. To view a copy of this license, visit http://creativecommons.org/ licenses/by/4.0/.

(c) The Author(s) 2020 